\title{
Hygrothermal effects on the buckling of soft-core sandwich plates with composite layered face sheets
}

\author{
S. Kamarian ${ }^{\mathrm{a}}$, M. Bodaghi ${ }^{\mathrm{b}}$, J. I. Song ${ }^{\mathrm{a}^{\dagger}}$ \\ a. Department of Mechanical Engineering, Changwon National University, Changwon, South Korea \\ b. Department of Engineering, School of Science and Technology, Nottingham Trent University, Nottingham, NG11 8NS, United Kingdom
}

\begin{abstract}
The present work attempts to investigate the influence of hygrothermal environments on the buckling of soft-core sandwich plates with laminated composite face sheets. The stability equations are derived based on piecewise low-order shear deformation theory (PLSDT) and then solved using different methods, depending on the boundary conditions. For fully simply supported sandwich plates, an analytical method is developed which results in some closed-form solutions. For sandwich plates with two parallel simply supported edges and two clamped edges, a semi-analytical solution is established using one-dimensional generalized differential quadrature (GDQ) technique. Finally, for fully clamped sandwich plates, twodimensional GDQ approach is applied to the governing equations. To validate the results of proposed methods, a comparative study with various examples is carried out verifying that the methods in the present work can accurately predict the buckling load of sandwich plates in hot and wet environments. Then, a comprehensive parametric study is performed to show the effects of temperature and moisture content on the buckling of sandwich plates for different parameters such as length to thickness ratio, core thickness and boundary conditions. It is concluded that both temperature and moisture content have significant influences on the buckling behavior of sandwich plates.
\end{abstract}

Keywords: Sandwich plates; Buckling; Hygrothermal environments; Analytical solution; GDQ method

\footnotetext{
${ }^{\dagger}$ Corresponding Author, E-mail: jisong@ @ changwon.ac.kr
} 


\section{Introduction}

Composite plates are used in most industries including marine, automotive, aerospace and construction due to their high strength, low weight, remarkable rigidity, high corrosion resistance and long fatigue life. When these structures are subjected to compressive loads, there is a possibility of buckling in them [1-10]. In many engineering applications, composite structures may be exposed to hot and wet environments. Since the hygrothermal environments play an important role on the stiffness of composite structures, the effects of two parameters of moisture and heat should be considered in the analysis of these structures [11-14].

Depending on the geometry of the structures and boundary conditions, moisture-induced swelling and heat-induced expansion can seriously affect the buckling phenomenon in composite structures [15]. The first research work in this area is probably Whitney and Ashton's study in which the effects of temperature and humidity of environment on the elastic response of laminated composited plates were investigated [16]. Based on classical plate theory (CPT), it was shown that swelling can significantly affect the stiffness of composite structures and therefore their bending, buckling and vibration. Flaggs and Vinson [17] developed a formulation for buckling phenomenon of general layered composite plates which extended the previous work [16]. The formulation included both hygrothermal and mechanical loadings and took into account the transverse shear and normal deformations. From this research work, it was found that hygrothermal environments can considerably decrease the in-plane mechanical loads necessary for buckling of composite structures. Therefore, it was also emphasized that the influence of temperature and moisture of environment should be considered in the analysis and design of composite structures. Ram and Sinha [18] studied free vibration and buckling of composite plates with cutout under hygrothermal environment. The researchers concluded that for thick plates, moisture has no significant effect on their buckling and vibration, but thin composite plates are highly affected by the moisture content. In another work, these researchers analyzed instability of layered composite plates exposed to moisture and heat [19]. The temperature and humiditydependent material properties were considered using experimental data. A parametric study was presented to show the influence of hygrothermal loads on the buckling of composite plates with respect to different geometric parameters, layups, boundary conditions, and temperature and moisture distributions. It was resulted that hot and wet environments can seriously change the stability of composite structures. The numerical results showed that uniform moisture concentration reduces critical buckling loads almost linearly. Concerning the role of temperature on the buckling of composite plates, it was concluded that at lower temperatures, the critical buckling load decreases almost linearly with increasing temperature, but at

high temperatures, this decrease is almost nonlinear. Chao and Shyu [20] studied non-linear buckling of laminated composite plates subjected to hygroscopic and thermal conditions. The formulation was provided 
for symmetric and unsymmetrical layups and then numerically handled for various boundary conditions. A parametrical study was performed to indicate the influence of fiber volume fraction, layups, geometric parameters, moisture content and temperature on the critical buckling load of the structure. The numerical results clarified that high temperature-humid environments can significantly reduce the buckling loads for plates. It was also found that with increasing moisture concentration, the mechanical buckling loads linearly decrease which is more pronounced in thin plates. Sreehari and Maiti [21] developed a finite element formulation to investigate the buckling and post-buckling of composite plates subjected to mechanical loads and hygrothermal environments. They examined the buckling response of the structure for different geometrical parameters, moisture content and temperature employing an inverse hyperbolic shear deformation theory. A conclusion was made that thermal and humid environments have a considerable effect on the buckling response of composite structures. Ghadirian et al. [22] studied vibration and stability of composite plates exposed to moisture and heat. They used trigonometric Ritz method to obtain the critical buckling temperature and moisture content, natural frequencies and mode shape for different types of boundary conditions. A comprehensive study was provided to show the effect of moisture-induced swelling and thermal expansions on the behavior of composite plates in buckling and vibration. Sreehari and Maiti [23] maximized buckling load of damaged layered composite plates under hygrothermal effects using particle swarm optimization (PSO) as one the most popular meta-heuristic algorithms for optimization problems. The orientations of fibers were considered as the design variables and a comparison was performed to evaluate the optimum results for a variety of aspect ratios and boundary conditions. Chen et al. [24] made a research to examine vibration and stability of initially stressed hybrid composite plates by considering the effect of hygrothermal environment. They provided a solution for composite plates with simply supported boundary conditions. From this study, it was concluded that buckling load is significantly affected by changes in humidity and temperature of environment. Amoushahi and Goodarzian [25] did a research work to demonstrate the influences of hygrothermal conditions on the dynamic and buckling response of composite plates with/ without strip delamination. The governing equations were obtained based on first-order shear deformation theory (FSDT) and solved using a finite strip method for different boundary conditions. The variations of critical buckling loads and natural frequencies of composite plates with respect to different configurations were thoroughly examined.

The unique characteristics of sandwich plates have led some researchers to study the thermal buckling of these types of structures. Babu and Kant $[26,27]$ employed two finite element formulations for thermal buckling analysis of sandwich plates with layered composite face sheets. They performed a parametric investigation to show the influences of different types of boundary conditions, thickness of layers and angle of fibers on the critical buckling temperature of the sandwich plates. They found that as the face sheets 
thicken, the buckling temperature of the structure decreases. Shiau and Kuo [28] analyzed thermal buckling of sandwich plates with laminated face sheets. It was inferred from this study that the optimum fiber orientation for a square sandwich plate with $( \pm \theta / \text { Core })_{s}$ face sheets is $+45^{\circ}$ while this amount for rectangular ones with aspect ratio greater than 2 is about $+63^{\circ}$. The researchers also concluded that composite materials with high $E_{1} / E_{2}$ and low $\alpha_{1} / \alpha_{2}$ ratios result in high critical buckling temperature. Matsunaga [29, 30] studied thermal buckling of rectangular cross-ply and angle-ply composite and sandwich plates using two-dimensional higher-order shear deformation theory (HSDT). In these studies, different truncated $m^{\text {th }}$-order theories were applied to obtain the buckling temperature of the sandwich plates. The accuracy of the results was evaluated in comparison with the results of three-dimensional layer wise solution. Vangipuram and Ganesan [31] conducted a research work to examine buckling and free vibrational behaviors of sandwich plates under thermal effects. It was observed that the coupling thermal conductivity term does not considerably affect thermal buckling of the structure. A decoupled thermomechanical analysis was presented by Pradeep and Ganesan [32] to investigate thermal buckling and free vibration of multi-layered viscoelastic sandwich plates. The researchers showed the variations of the critical buckling temperatures, natural frequencies and mode shapes against various effective parameters like core thickness. Han et al. [33] developed a new, accurate and simple refined theory to examine buckling and free vibration of foam-filled corrugated rectangular sandwich plates with simply supported boundary conditions considering thermal influence of environment. They showed that foam filling can decrease the performance of the structure in buckling and vibration up to $13.7 \%$. Based on the sublaminate generalized unified formulation, Vescovini et al. [34] studied thermal buckling behavior of sandwich plates. The proficiency of the applied approach was evaluated by comparing the results with three-dimensional solution. Zhai et al. [35] investigated buckling temperature and natural frequencies of simply supported soft-core sandwich plates with single-layer orthotropic skins under thermal environments effects. They studied the buckling and vibration characteristics of sandwich plates analytically and concluded that they are significantly affected by temperature-induced thermal stresses.

Sandwich plates with polymer composite face sheets are widely used for aerospace and marine structures. They may usually be subjected to hygroscopic and thermal environments during their service [36, 37]. Although the previous researches indicate that hot and wet environments can dramatically affect the stability performance of composite structures, to the best of authors' knowledge, no research work has been performed on the buckling of sandwich plates by considering the simultaneous effects of humidity and temperature, or the number of these studies is very limited. Due to the lack of a comprehensive survey in this field of research, the present work is motivated to focus on the buckling response of sandwich plates 
with soft cores and composite layered face sheets in hygrothermal environments. Since the exact analysis of this type of structures is very complicated and time consuming, a straightforward solution with high performance should be sought that can simplify the formulation of the problem and reduce the computational time and cost as much as possible. To this end, an appropriate stability formulation for sandwich plates under hygro-thermo-mechanical loads is developed here based on PLSDT [38]. The use of this theory has a complexity, which is the inequality of the number of governing equations and boundary conditions. For this reason, depending on the type of boundary conditions, the stability equations are solved using different methods of analytical, semi analytical and numerical techniques. For fully simply supported sandwich plates, an analytical method is applied and some close-form solutions are provided for estimation of critical buckling loads. For the case where the structure has two opposite simply supported and two opposite clamped boundary conditions, a semi analytical method is utilized using one-dimensional GDQ technique, as an efficient and powerful tool for solving partial differential equations [39-42]. Finally, for fully clamped sandwich plates, two-dimensional GDQ approach is implemented for the buckling analysis. Then, the influences of moisture content and temperature of environments on the critical buckling load of sandwich plates is comprehensively studied for different geometrical parameters and boundary conditions.

\section{Problem description}

\subsection{Thermal buckling equations}

Consider a rectangular sandwich plate of dimension $a \times b \times h$, as shown in Fig. 1. The sandwich plate is assumed to be under compressive in-plane forces and exposed to hygrothermal environment. The thicknesses of each face sheet and core are $h_{f}$ and $2 h_{c}$, respectively. Though the cores of sandwich structures are usually non-composite or non-laminated, a general formulation is provided here for sandwich plates consisting of laminated composite cores and face sheets. Based on PLSDT [38], the displacement fields for the structure can be expressed as:

Transverse displacement component at any point:

$$
w(x, y, z)=w(x, y),
$$

In-plane displacement components
in the top and bottom face sheets: $\left\{\begin{array}{l}u_{t}(x, y, z)=u_{0}(x, y) \mp \frac{2 h_{c}+h_{f}}{2} \psi_{1}-\left(z \mp \frac{2 h_{c}+h_{f}}{2}\right) \frac{\partial w}{\partial x} \\ v_{t}(x, y, z)=v_{0}(x, y) \mp \frac{2 h_{c}+h_{f}}{2} \psi_{2}-\left(z \mp \frac{2 h_{c}+h_{f}}{2}\right) \frac{\partial w}{\partial y}\end{array}\right.$ 
In plane displacement components
in the core: $\left\{\begin{array}{l}u_{c}(x, y, z)=u_{0}(x, y)-z\left(\frac{2 h_{c}+h_{f}}{2 h_{c}} \psi_{1}-\frac{h_{f}}{2 h_{c}} \frac{\partial w}{\partial x}\right) \\ v_{c}(x, y, z)=v_{0}(x, y)-z\left(\frac{2 h_{c}+h_{f}}{2 h_{c}} \psi_{2}-\frac{h_{f}}{2 h_{c}} \frac{\partial w}{\partial y}\right)\end{array}\right.$

where index $c$ denotes the core layer, and indices $t$ and $b$ represent the upper and lower face sheets, respectively. Moreover, $\psi_{1}$ and $\psi_{2}$ are the rotation angles of the straight line that connects the midpoint of the face sheets in the $x o z$ and yoz planes, respectively. Considering Strain von-Karman's nonlinearity terms, the strains of the face sheets can be written as:

$$
\begin{aligned}
& \varepsilon_{t x}=\frac{\partial u_{t}}{\partial x}+\frac{1}{2}\left(\frac{\partial w}{\partial x}\right)^{2}=\left(\frac{\partial u_{0}}{\partial x} \mp \frac{2 h_{c}+h_{f}}{2} \frac{\partial \psi_{1}}{\partial x}+\frac{1}{2}\left(\frac{\partial w}{\partial x}\right)^{2}\right)+\left(z \mp \frac{2 h_{c}+h_{f}}{2}\right)\left(-\frac{\partial^{2} w}{\partial x^{2}}\right)={\stackrel{(0)}{\varepsilon_{b} x}}_{b}+\left(z \mp \frac{2 h_{c}+h_{f}}{2}\right)^{(1)} \varepsilon_{b^{x}}^{(x)} \\
& \varepsilon_{t, y}=\frac{\partial v_{t}}{\partial y}+\frac{1}{2}\left(\frac{\partial w}{\partial y}\right)^{2}=\left(\frac{\partial v_{0}}{\partial y} \mp \frac{2 h_{c}+h_{f}}{2} \frac{\partial \psi_{2}}{\partial y}+\frac{1}{2}\left(\frac{\partial w}{\partial y}\right)^{2}\right)+\left(z \mp \frac{2 h_{c}+h_{f}}{2}\right)\left(-\frac{\partial^{2} w}{\partial y^{2}}\right)={\stackrel{(0)}{\varepsilon_{b}, y}}_{b}+\left(z \mp \frac{2 h_{c}+h_{f}}{2}\right)_{\varepsilon_{b},}^{(1)} \\
& \gamma_{t x y}=\frac{\partial u_{t}}{\partial y}+\frac{\partial v_{t}}{\partial x}=\left(\left(\frac{\partial u_{0}}{\partial y}+\frac{\partial v_{0}}{\partial x}\right) \mp\left(\frac{2 h_{c}+h_{f}}{2}\right)\left(\frac{\partial \psi_{1}}{\partial y}+\frac{\partial \psi_{2}}{\partial x}\right)\right)+\left(z \mp \frac{2 h_{c}+h_{f}}{2}\right)\left(-2 \frac{\partial^{2} w}{\partial x \partial y}\right)=\gamma_{t, y}^{(0)}+\left(z \mp \frac{2 h_{c}+h_{f}}{2}\right) \gamma_{b, y}^{(1)}
\end{aligned}
$$

Similarly, the strain components for the core can be obtained in the following forms:

$$
\begin{aligned}
& \varepsilon_{c x}=\frac{\partial u_{c}}{\partial x}+\frac{1}{2}\left(\frac{\partial w}{\partial x}\right)^{2}=\left(\frac{\partial u_{0}}{\partial x}+\frac{1}{2}\left(\frac{\partial w}{\partial x}\right)^{2}\right)+z\left(-\frac{2 h_{c}+h_{f}}{2 h_{c}} \frac{\partial \psi_{1}}{\partial x}+\frac{h_{f}}{2 h_{c}} \frac{\partial^{2} w}{\partial x^{2}}\right)=\stackrel{(}{\varepsilon}_{c x}^{(0)}+z{\stackrel{(1)}{\varepsilon_{c x}}}^{2} \\
& \varepsilon_{c y}=\frac{\partial v_{c}}{\partial y}+\frac{1}{2}\left(\frac{\partial w}{\partial y}\right)^{2}=\left(\frac{\partial v_{0}}{\partial y}+\frac{1}{2}\left(\frac{\partial w}{\partial y}\right)^{2}\right)+z\left(-\frac{2 h_{c}+h_{f}}{2 h_{c}} \frac{\partial \psi_{2}}{\partial y}+\frac{h_{f}}{2 h_{c}} \frac{\partial^{2} w}{\partial y^{2}}\right)=\stackrel{(0)}{\varepsilon}_{c y}+z{\stackrel{(1)}{\varepsilon_{c}}}^{(1)} \\
& \gamma_{c x y}=\frac{\partial u_{c}}{\partial y}+\frac{\partial v_{c}}{\partial x}=\left(\frac{\partial u_{0}}{\partial y}+\frac{\partial v_{0}}{\partial x}\right)+z\left(-\frac{2 h_{c}+h_{f}}{2 h_{c}}\left(\frac{\partial \psi_{1}}{\partial y}+\frac{\partial \psi_{2}}{\partial x}\right)+\frac{h_{f}}{2 h_{c}}\left(2 \frac{\partial^{2} w}{\partial x \partial y}\right)\right)=\gamma_{c x y}^{(0)}+z \gamma_{c x y}^{(1)} \\
& \gamma_{c x z}=\frac{\partial u_{c}}{\partial z}+\frac{\partial w}{\partial x}=\frac{2 h_{c}+h_{f}}{2 h_{c}}\left(\frac{\partial w}{\partial x}-\psi_{1}\right) \\
& \gamma_{c y z}=\frac{\partial v_{c}}{\partial z}+\frac{\partial w}{\partial y}=\frac{2 h_{c}+h_{f}}{2 h_{c}}\left(\frac{\partial w}{\partial y}-\psi_{2}\right)
\end{aligned}
$$

For the sandwich plate under hygrothermal environments, the stress components in the $k^{\text {th }}$ layer of the face sheets or core can be calculated in the forms of:

$$
\begin{aligned}
& \left\{\begin{array}{c}
\sigma_{t x} \\
b \\
\sigma_{t y}^{k t} \\
b \\
\tau_{t x y} \\
b
\end{array}\right\}^{k t}=\left[\begin{array}{lll}
\bar{Q}_{11} & \bar{Q}_{12} & \bar{Q}_{16} \\
& \bar{Q}_{22} & \bar{Q}_{26}^{k t} \\
S y m & & \bar{Q}_{66}
\end{array}\right]^{b}\left(\left\{\begin{array}{c}
\varepsilon_{t x} \\
b \\
\varepsilon_{t y} \\
b \\
\gamma_{t x y} \\
b
\end{array}\right\}^{k t}-\Delta T\left\{\begin{array}{c}
\alpha_{t x} \\
\alpha_{t y} \\
b \\
2 \alpha_{t x y} \\
b
\end{array}\right\}^{k t}-\Delta C\left\{\begin{array}{c}
\beta_{t x} \\
b \\
\beta_{t y} \\
b \\
2 \beta_{t x y} \\
b
\end{array}\right\}^{k t} b\right. \\
& \left\{\begin{array}{l}
\sigma_{c x} \\
\sigma_{c y} \\
\tau_{c x y}
\end{array}\right\}^{k c}=\left[\begin{array}{lll}
\bar{Q}_{11} & \bar{Q}_{12} & \bar{Q}_{16} \\
& \bar{Q}_{22} & \bar{Q}_{26} \\
S y m & \bar{Q}_{66}
\end{array}\right]^{k c}\left(\left\{\begin{array}{c}
\varepsilon_{c x} \\
\varepsilon_{c y} \\
\gamma_{c x}
\end{array}\right\}^{k c}-\Delta T\left\{\begin{array}{l}
\alpha_{c x} \\
\alpha_{c y} \\
2 \alpha_{c x y}
\end{array}\right\}^{k c}-\Delta M\left\{\begin{array}{l}
\beta_{c x} \\
\beta_{c y} \\
2 \beta_{c x y}
\end{array}\right\}\right) \\
& \left\{\begin{array}{l}
\tau_{c y z} \\
\tau_{c x z}
\end{array}\right\}^{k c}=\left[\begin{array}{ll}
\bar{Q}_{44} & \bar{Q}_{45} \\
\bar{Q}_{45} & \bar{Q}_{55}
\end{array}\right]\left\{\begin{array}{l}
\gamma_{c y z} \\
\gamma_{c x z}
\end{array}\right\}^{k c}
\end{aligned}
$$


where $\bar{Q}_{i j}$ denotes the transformed reduced stiffness coefficients detailed in Appendix $1 ; \alpha$ and $\beta$ represent the thermal expansion coefficient and moisture expansion coefficient described in Appendix 2; $\Delta T$ and $\Delta C$ are the temperature and moisture changes with respect to ideal environment (room temperature without moisture content), and $k t, k b$ and $k c$ refer to the $k^{\text {th }}$ layer in top face, bottom face and the core, respectively. It should be mentioned that some assumptions are taken into consideration in the present work that are:

- The top and bottom face sheets are similar.

- The layups of face sheets or core are cross-ply $\left(\bar{Q}_{16}=\bar{Q}_{26}=\bar{Q}_{45}=0\right)$ and symmetric with respect to their mid-planes.

- The temperature and moisture are uniformly distributed.

- The core and face sheets are considered to be isotropic or orthotropic.

By using the above assumptions, the stress resultants in the face sheets can be defined as:

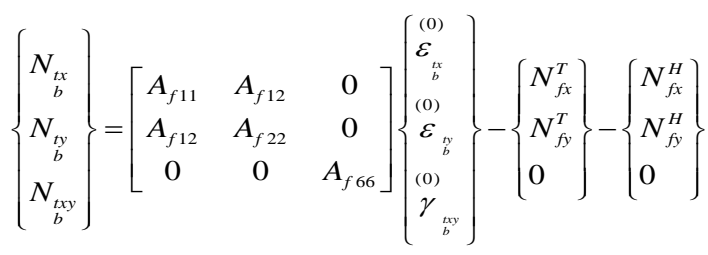

$$
\begin{aligned}
& \left\{\begin{array}{c}
M_{t x} \\
b \\
M_{t y} \\
b \\
M_{t x y} \\
b
\end{array}\right\}=\left[\begin{array}{ccc}
D_{f 11} & D_{f 12} & 0 \\
D_{f 12} & D_{f 22} & 0 \\
0 & 0 & D_{f 66}
\end{array}\right]\left\{\begin{array}{c}
(1) \\
\mathcal{E}_{t z} \\
(1) \\
\mathcal{E}_{b y} \\
b \\
(1) \\
\gamma_{t a y} \\
b y
\end{array}\right\}
\end{aligned}
$$

where $A_{f i j}$ and $D_{f i j}$ are the extensional and bending stiffness coefficients for the skins, respectively, defined as:

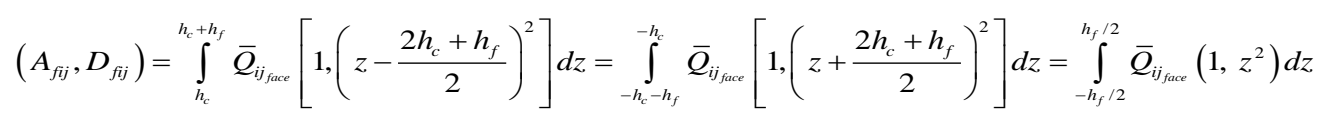

In Eq. (5), $N_{f x}^{T}$ and $N_{f y}^{T}$ denote the in-plane thermal forces, and $N_{f x}^{H}$ and $N_{f y}^{H}$ represent the in-plane hygroscopic forces in the face sheets calculated by the following relations:

$$
\begin{aligned}
& \left\{\begin{array}{l}
N_{f x}^{T} \\
N_{f y}^{T}
\end{array}\right\}=\left\{\begin{array}{l}
N_{t x}^{T} \\
N_{t y}^{T}
\end{array}\right\}=\left\{\begin{array}{l}
N_{b x}^{T} \\
N_{b y}^{T}
\end{array}\right\}=\int_{-h_{f} / 2}^{h_{f} / 2}\left[\begin{array}{ll}
\bar{Q}_{11} & \bar{Q}_{12} \\
\bar{Q}_{12} & \bar{Q}_{22}
\end{array}\right]_{\text {face }}\left\{\begin{array}{l}
\alpha_{x} \\
\alpha_{y}
\end{array}\right\}_{\text {face }} \Delta T d z \\
& \left\{\begin{array}{l}
N_{f x}^{H} \\
N_{f y}^{H}
\end{array}\right\}=\left\{\begin{array}{l}
N_{t x}^{H} \\
N_{t y}^{H}
\end{array}\right\}=\left\{\begin{array}{l}
N_{b x}^{H} \\
N_{b y}^{H}
\end{array}\right\}=\int_{-h_{f} / 2}^{h_{f} / 2}\left[\begin{array}{ll}
\bar{Q}_{11} & \bar{Q}_{12} \\
\bar{Q}_{12} & \bar{Q}_{22}
\end{array}\right]_{\text {face }}\left\{\begin{array}{l}
\beta_{x} \\
\beta_{y}
\end{array}\right\}_{\text {face }} \Delta C d z
\end{aligned}
$$

Also, the stress resultants for the core can be defined as: 


$$
\begin{aligned}
& \left\{\begin{array}{l}
N_{c x} \\
N_{c y} \\
N_{c x y}
\end{array}\right\}=\left[\begin{array}{ccc}
A_{c 11} & A_{c 12} & 0 \\
& A_{c 22} & 0 \\
S y m & & A_{c 66}
\end{array}\right]\left\{\begin{array}{l}
(0) \\
\varepsilon_{c x} \\
(0) \\
\mathcal{E}_{c y} \\
(0) \\
\gamma_{c x y}
\end{array}\right\}-\left\{\begin{array}{l}
N_{c x}^{T} \\
N_{c y}^{T} \\
0
\end{array}\right\}-\left\{\begin{array}{l}
N_{c x}^{H} \\
N_{c y}^{H} \\
0
\end{array}\right\} \\
& \left\{\begin{array}{l}
M_{c x} \\
M_{c y} \\
M_{c x y}
\end{array}\right\}=\left[\begin{array}{ccc}
D_{c 11} & D_{c 12} & 0 \\
& D_{c 22} & 0 \\
S y m & & D_{c 66}
\end{array}\right]\left\{\begin{array}{l}
(1) \\
\varepsilon_{c x} \\
(1) \\
\varepsilon_{c y} \\
(1) \\
\gamma_{c x y}
\end{array}\right\} \\
& \left\{\begin{array}{l}
Q_{c y z} \\
Q_{c x z}
\end{array}\right\}=\left[\begin{array}{cc}
A_{c 44} & 0 \\
0 & A_{c 55}
\end{array}\right]\left\{\begin{array}{l}
\gamma_{c y z} \\
\gamma_{c x z}
\end{array}\right\}
\end{aligned}
$$

in which,

$$
\begin{aligned}
& \left(A_{c i j}, D_{c i j}\right)=\int_{-h_{c}}^{h_{c}} \bar{Q}_{i j_{c o r e}}\left(1, z^{2}\right) d z \\
& \left\{\begin{array}{l}
N_{c x}^{T} \\
N_{c y}^{T}
\end{array}\right\}=\int_{-h_{c}}^{h_{c}}\left[\begin{array}{ll}
\bar{Q}_{11} & \bar{Q}_{12} \\
\bar{Q}_{12} & \bar{Q}_{22}
\end{array}\right]_{c o r e}\left\{\begin{array}{l}
\alpha_{x} \\
\alpha_{y}
\end{array}\right\}_{\text {core }} \Delta T d z \\
& \left\{\begin{array}{l}
N_{c x}^{H} \\
N_{c y}^{H}
\end{array}\right\}=\int_{-h_{c}}^{h_{c}}\left[\begin{array}{ll}
\bar{Q}_{11} & \bar{Q}_{12} \\
\bar{Q}_{12} & \bar{Q}_{22}
\end{array}\right]_{\text {core }}\left\{\begin{array}{l}
\beta_{x} \\
\beta_{y}
\end{array}\right\}_{\text {core }} \Delta C d z
\end{aligned}
$$

In the next step, the strain energy $(U)$ of the sandwich plate is calculated as:

$$
\begin{aligned}
& U=U_{t}+U_{b}+U_{c} \\
& U_{t}=U_{t x}+U_{t y}+U_{t x y}=\frac{1}{2} \int_{0}^{a} \int_{0}^{b} \int_{0}^{h_{c}+h_{f}} \int_{h_{c}} \sigma_{t x} \varepsilon_{t x} d z d y d x+\frac{1}{2} \int_{0}^{a} \int_{0}^{b} \int_{0}^{h_{c}+h_{f}} \int_{h_{c}} \sigma_{t y} \varepsilon_{t y} d z d y d x+\int_{0}^{a} \int_{0}^{b} \int_{h_{c}}^{h_{c}+h_{f}} \tau_{t x y} \gamma_{t x y} d z d y d x \\
& U_{b}=U_{b x}+U_{b y}+U_{b x y}=\frac{1}{2} \int_{0}^{a} \int_{0}^{b} \int_{-h_{c}-h_{f}}^{h_{c}} \sigma_{b x} \varepsilon_{b x} d z d y d x+\frac{1}{2} \int_{0}^{a} \int_{0}^{b} \int_{-h_{c}-h_{f}}^{h_{c}} \sigma_{b y} \varepsilon_{b y} d z d y d x+\int_{0}^{a} \int_{0}^{b} \int_{-h_{c}-h_{f}}^{-h_{c}} \tau_{b x y} \gamma_{b x y} d z d y d x \\
& U_{c}=U_{c x}+U_{c y}+U_{c x y}+U_{c x z}+U_{c y z}=\frac{1}{2} \int_{0}^{a} \int_{0}^{b} \int_{-h_{c}}^{h_{c}} \sigma_{c x} \varepsilon_{c x} d z d y d x+\frac{1}{2} \int_{0}^{b} \int_{0}^{h_{c}} \int_{-h_{c}}^{h_{c}} \sigma_{c y} \varepsilon_{c y} d z d y d x \\
& +\int_{0}^{a} \int_{0}^{b} \int_{-h_{c}}^{h_{c}} \tau_{c x y} \gamma_{c x y} d z d y d x+\int_{0}^{a} \int_{0}^{b} \int_{-h_{c}}^{h_{c}} \tau_{c x z} \gamma_{c x z} d z d y d x+\int_{0}^{a} \int_{0}^{b} \int_{-h_{c}}^{h_{c}} \tau_{c y z} \gamma_{c y z} d z d y d x
\end{aligned}
$$

The work done by the external compressive loads $\left(P_{x}\right.$ and $\left.P_{y}\right)$ can be derived by:

$$
W=-\frac{1}{2} \int_{0}^{b} \int_{0}^{a}\left[P_{x}\left(\frac{\partial w}{\partial x}\right)^{2}+P_{y}\left(\frac{\partial w}{\partial y}\right)^{2}\right] d x d y
$$

Based on the principle of virtual work [43], we have:

$$
\delta(U+W)=0
$$

By inserting Eqs. (10) and (11) into Eq. (12), five governing equations are obtained, two of which relate to in-plane equations and the remaining three equations relate to out of plane equations. By implementing adjacent equilibrium buckling criterion, performing pre-buckling analysis for sandwich plates with immovable simply supported and clamped boundary conditions, as well as linearization of the obtained 
relations, the thermal buckling equations of the structure in terms of displacement components can be expressed as:

$$
\begin{aligned}
& g_{11}^{(3)} \frac{\partial^{4} w}{\partial x^{4}}+\left(2 g_{12}^{(3)}+4 g_{66}^{(3)}\right) \frac{\partial^{4} w}{\partial x^{2} \partial y^{2}}+g_{22}^{(3)} \frac{\partial^{4} w}{\partial y^{4}}+g_{11}^{(2)} \frac{\partial^{3} \psi_{1}}{\partial x^{3}}+\left(g_{12}^{(2)}+2 g_{66}^{(2)}\right)\left(\frac{\partial^{3} \psi_{1}}{\partial x \partial y^{2}}+\frac{\partial^{3} \psi_{2}}{\partial x^{2} \partial y}\right)+ \\
& g_{22}^{(2)} \frac{\partial^{3} \psi_{2}}{\partial y^{3}}+S_{c x z}\left(\frac{\partial \psi_{1}}{\partial x}-\frac{\partial^{2} w}{\partial x^{2}}\right)+S_{c y z}\left(\frac{\partial \psi_{2}}{\partial y}-\frac{\partial^{2} w}{\partial y^{2}}\right)+\left(N_{x}^{T}+N_{x}^{M}+P_{x}\right) \frac{\partial^{2} w}{\partial x^{2}}+\left(N_{y}^{T}+N_{y}^{M}+P_{y}\right) \frac{\partial^{2} w}{\partial y^{2}}=0 \\
& -g_{11}^{(2)} \frac{\partial^{3} w}{\partial x^{3}}-\left(g_{12}^{(2)}+2 g_{66}^{(2)}\right) \frac{\partial^{3} w}{\partial x \partial y^{2}}-\left(g_{12}^{(1)}+g_{66}^{(1)}\right) \frac{\partial^{2} \psi_{2}}{\partial x \partial y}-g_{11}^{(1)} \frac{\partial^{2} \psi_{1}}{\partial x^{2}}-g_{66}^{(1)} \frac{\partial^{2} \psi_{1}}{\partial y^{2}}+S_{c x z}\left(\psi_{1}-\frac{\partial w}{\partial x}\right)=0 \\
& -g_{22}^{(2)} \frac{\partial^{3} w}{\partial y^{3}}-\left(g_{12}^{(2)}+2 g_{66}^{(2)}\right) \frac{\partial^{3} w}{\partial x^{2} \partial y}-\left(g_{12}^{(1)}+g_{66}^{(1)}\right) \frac{\partial^{2} \psi_{1}}{\partial x \partial y}-g_{22}^{(1)} \frac{\partial^{2} \psi_{2}}{\partial y^{2}}-g_{66}^{(1)} \frac{\partial^{2} \psi_{2}}{\partial x^{2}}+S_{c y z}\left(\psi_{2}-\frac{\partial w}{\partial y}\right)=0
\end{aligned}
$$

in which

$$
\begin{aligned}
& N_{x}^{T}=N_{c x}^{T}+N_{t x}^{T}+N_{b x}^{T}=N_{c x}^{T}+2 N_{f x}^{T} \\
& N_{x}^{H}=N_{c x}^{H}+N_{t x}^{H}+N_{b x}^{H}=N_{c x}^{H}+2 N_{f x}^{H} \\
& S_{c x z}=\left(1+\frac{2 h_{c}+h_{f}}{2 h_{c}}\right)^{2} A_{c 55} \\
& S_{c y z}=\left(1+\frac{2 h_{c}+h_{f}}{2 h_{c}}\right)^{2} A_{c 44}
\end{aligned}
$$

and the coefficients of $g_{i j}^{(k)}$ are presented in Appendix 3. The related boundary conditions can also be derived as:

$$
\begin{aligned}
x=0, a: \begin{cases}\delta w=0 & \text { or }\left(-g_{11}^{(2)} \frac{\partial^{2} \psi_{1}}{\partial x^{2}}-g_{11}^{(3)} \frac{\partial^{3} w}{\partial x^{3}}-g_{12}^{(2)} \frac{\partial^{2} \psi_{2}}{\partial x \partial y}-g_{12}^{(3)} \frac{\partial^{3} w}{\partial x \partial y^{2}}+S_{c x z}\left(\frac{\partial w}{\partial x}-\psi_{1}\right)\right)=0 \\
\delta\left(\frac{\partial w}{\partial x}\right)=0 & \text { or }\left(g_{11}^{(2)} \frac{\partial \psi_{1}}{\partial x}+g_{11}^{(3)} \frac{\partial^{2} w}{\partial x^{2}}+g_{12}^{(2)} \frac{\partial \psi_{2}}{\partial y}+g_{12}^{(3)} \frac{\partial^{2} w}{\partial y^{2}}\right)=0 \\
\delta \psi_{1}=0 & \text { or }\left(g_{11}^{(1)} \frac{\partial \psi_{1}}{\partial x}+g_{11}^{(2)} \frac{\partial^{2} w}{\partial x^{2}}+g_{12}^{(1)} \frac{\partial \psi_{2}}{\partial y}+g_{12}^{(2)} \frac{\partial^{2} w}{\partial y^{2}}\right)=0\end{cases} \\
y=0, b: \begin{cases}\delta w=0 & \text { or }\left(-g_{22}^{(2)} \frac{\partial^{2} \psi_{2}}{\partial y^{2}}-g_{22}^{(3)} \frac{\partial^{3} w}{\partial y^{3}}-g_{12}^{(2)} \frac{\partial^{2} \psi_{1}}{\partial x \partial y}-g_{12}^{(3)} \frac{\partial^{3} w}{\partial x^{2} \partial y}+S_{c y z}\left(\frac{\partial w}{\partial y}-\psi_{2}\right)\right)=0 \\
\delta\left(\frac{\partial w}{\partial y}\right)=0 & \text { or }\left(g_{22}^{(2)} \frac{\partial \psi_{2}}{\partial y}+g_{22}^{(3)} \frac{\partial^{2} w}{\partial y^{2}}+g_{12}^{(2)} \frac{\partial \psi_{1}}{\partial x}+g_{12}^{(3)} \frac{\partial^{2} w}{\partial x^{2}}\right)=0 \\
\delta \psi_{2}=0 & \text { or }\left(g_{22}^{(1)} \frac{\partial \psi_{2}}{\partial y}+g_{22}^{(2)} \frac{\partial^{2} w}{\partial y^{2}}+g_{12}^{(1)} \frac{\partial \psi_{1}}{\partial x}+g_{12}^{(2)} \frac{\partial^{2} w}{\partial x^{2}}\right)=0\end{cases}
\end{aligned}
$$

It is worth noting that for the clamped boundary conditions, we have $\delta w=\delta\left(\frac{\partial w}{\partial x}\right)=\delta\left(\frac{\partial w}{\partial y}\right)=\delta \psi_{1}=\delta \psi_{2}=0$, thus, Eqs. (15) are automatically satisfied.

\subsection{Solution methods}


This subsection attempts to explain how to solve thermal buckling equations of the rectangular sandwich plate with different boundary conditions, as presented in Eqs. (13) and (15). Here, depending on the type of boundaries, three different methods are provided.

\section{Simply supported boundary conditions (SSSS)}

For sandwich plates with simply supported edges, an analytical solution is suggested in the form of:

$$
\begin{aligned}
& w(x, y)=W_{m n} \sin \frac{m \pi}{a} x \sin \frac{n \pi}{b} y \\
& \psi_{1}(x, y)=\Psi_{1 m n} \cos \frac{m \pi}{a} x \sin \frac{n \pi}{b} y \\
& \psi_{2}(x, y)=\Psi_{2 m n} \sin \frac{m \pi}{a} x \cos \frac{n \pi}{b} y
\end{aligned}
$$

where $m$ and $n$ are the number of half-wave in $x$ - and $y$-directions. As can be observed, the purposed solution can satisfy the boundary conditions of Eq. (15). Inserting Eq. (16) into Eq. (13) results in:

$$
\left[\begin{array}{lll}
k_{11} & k_{12} & k_{13} \\
k_{21} & k_{22} & k_{23} \\
k_{31} & k_{32} & k_{33}
\end{array}\right]\left[\begin{array}{l}
W_{m n} \\
\Psi_{1 m n} \\
\Psi_{2 m n}
\end{array}\right]=\left[\begin{array}{l}
0 \\
0 \\
0
\end{array}\right]
$$

in which the coefficients of $k_{i j}$ are expressed in Appendix 4. Then, a nontrivial solution is sought for the above equations as follows:

$$
\operatorname{det}\left[\begin{array}{lll}
k_{11} & k_{12} & k_{13} \\
k_{21} & k_{22} & k_{23} \\
k_{31} & k_{32} & k_{33}
\end{array}\right]=0
$$

By defining parameter $\lambda$ as the load ratio $\left(\lambda=\frac{P_{y}}{P_{x}}\right)$, solving Eq. (18) straightforwardly yields the buckling load in the following form.

$$
P_{x}=\frac{f_{1}-f_{2}}{f_{3}}
$$

where

$$
\begin{aligned}
& f_{1}=\left(\frac{m \pi}{a}\right)^{4} g_{11}^{(3)}+\left(\frac{m \pi}{a}\right)^{2}\left(\frac{n \pi}{b}\right)^{2}\left(2 g_{12}^{(3)}+4 g_{66}^{(3)}\right)+\left(\frac{n \pi}{b}\right)^{4} g_{22}^{(3)}+\left(\frac{m \pi}{a}\right)^{2} S_{c x z}+\left(\frac{n \pi}{b}\right)^{2} S_{c y z} \\
& f_{2}=f^{*}+\left(\frac{m \pi}{a}\right)^{2}\left(N_{x}^{T}+N_{x}^{H}\right)+\left(\frac{n \pi}{b}\right)^{2}\left(N_{y}^{T}+N_{y}^{H}\right) \\
& f_{3}=\left(\frac{m \pi}{a}\right)^{2}+\left(\frac{n \pi}{b}\right)^{2} \lambda \\
& f^{*}=\left(k_{12}^{2} k_{33}+k_{13}^{2} k_{22}-2 k_{12} k_{13} k_{23}\right) /\left(k_{22} k_{33}-k_{23}^{2}\right)
\end{aligned}
$$

From Eq. (19), the buckling loads of the structure are calculated for different values of $m$ and $n$. The minimum obtained value is considered as the critical buckling load signified by the symbol of $P_{c r}$. It should 
be noted that in the absence of external mechanical loads, this is also possible for composite plates to buckle due to only hygroscopic or thermal loads which is called hygrothermal buckling. In this case, for a specified amount of moisture $(\Delta C=c t e)$, the buckling temperatures can be obtained as:

$$
\Delta T=\frac{f_{1}-f_{4}}{f_{5}}
$$

where

$$
\begin{aligned}
& f_{4}=f^{*}+\left(\frac{m \pi}{a}\right)^{2} N_{x}^{H}+\left(\frac{n \pi}{b}\right)^{2} N_{y}^{H} \\
& f_{5}=\left(\frac{m \pi}{a}\right)^{2} \tilde{N}_{x}^{T}+\left(\frac{n \pi}{b}\right)^{2} \tilde{N}_{y}^{T} \\
& \left(\begin{array}{c}
\tilde{N}_{x}^{T} \\
\tilde{N}_{y}^{T}
\end{array}\right)=\left(\begin{array}{c}
\tilde{N} c_{x}^{T} \\
\tilde{N} c_{y}^{T}
\end{array}\right)+2\left(\begin{array}{c}
\tilde{N} f_{x}^{T} \\
\tilde{N} f_{y}^{T}
\end{array}\right)=\int_{-h_{c}}^{+h_{c}}\left[\begin{array}{ll}
\bar{Q}_{11} & \bar{Q}_{12} \\
\bar{Q}_{12} & \bar{Q}_{22}
\end{array}\right]_{c o r e}\left\{\begin{array}{c}
\alpha_{x} \\
\alpha_{y}
\end{array}\right\}_{c o r e} d z+2 \int_{-h_{f} / 2}^{+h_{h} / 2}\left[\begin{array}{cc}
\bar{Q}_{11} & \bar{Q}_{12} \\
\bar{Q}_{12} & \bar{Q}_{22}
\end{array}\right]_{\text {face }}\left\{\begin{array}{l}
\alpha_{x} \\
\alpha_{y}
\end{array}\right\}_{\text {face }} d z
\end{aligned}
$$

Again, the critical buckling temperature $\left(\Delta T_{c r}\right)$ is the lowest value obtained for all $m$ and $n$. Similarly, in the absence of external load and at a certain temperature $(\Delta T=c t e)$, the buckling moisture contents can be obtain using the following equation:

$$
\Delta C=\frac{f_{1}-f_{6}}{f_{7}}
$$

where

$$
\begin{aligned}
& f_{6}=f^{*}+\left(\frac{m \pi}{a}\right)^{2} N_{x}^{T}+\left(\frac{n \pi}{b}\right)^{2} N_{y}^{T} \\
& f_{7}=\left(\frac{m \pi}{a}\right)^{2} \tilde{N}_{x}^{H}+\left(\frac{n \pi}{b}\right)^{2} \tilde{N}_{y}^{H} \\
& \left(\begin{array}{l}
\tilde{N}_{x}^{H} \\
\tilde{N}_{y}^{H}
\end{array}\right)=\left(\begin{array}{l}
\tilde{N} c_{x}^{H} \\
\tilde{N} c_{y}^{H}
\end{array}\right)+2\left(\begin{array}{l}
\tilde{N} f_{x}^{H} \\
\tilde{N} f_{y}^{H}
\end{array}\right)=\int_{-h_{c}}^{+h_{c}}\left[\begin{array}{ll}
\bar{Q}_{11} & \bar{Q}_{12} \\
\bar{Q}_{12} & \bar{Q}_{22}
\end{array}\right]_{c o r e}\left\{\begin{array}{l}
\beta_{x} \\
\beta_{y}
\end{array}\right\}_{\text {core }} d z+2 \int_{-h_{f} / 2}^{+h_{f} / 2}\left[\begin{array}{ll}
\bar{Q}_{11} & \bar{Q}_{12} \\
\bar{Q}_{12} & \bar{Q}_{22}
\end{array}\right]_{\text {face }}\left\{\begin{array}{l}
\beta_{x} \\
\beta_{y}
\end{array}\right\}_{\text {face }} d z
\end{aligned}
$$

and the lowest value for different $m$ and $n$ is known as the critical moisture content $\left(\Delta C_{c r}\right)$.

\section{Two opposite simply supported edges and two clamped edges (CSCS)}

For this case, the boundary conditions are assumed to be clamped at $x=0, a$ but simply supported at $y=0, b$. For sandwich plates with this type of boundaries, a semi analytical solution is presented. It can be seen that by adopting the following form for the displacement components, the boundary conditions at $y=0, b$ are satisfied. 


$$
\begin{aligned}
& w(x, y)=W(x) \sin \frac{n \pi}{b} y \\
& \psi_{1}(x, y)=\Psi_{1}(x) \sin \frac{n \pi}{b} y \\
& \psi_{2}(x, y)=\Psi_{2}(x) \cos \frac{n \pi}{b} y
\end{aligned}
$$

Substituting Eq. (25) into Eq. (13) leads to the following equations:

$$
\begin{aligned}
& p_{1} \frac{\partial^{4} W}{\partial x^{4}}+p_{2} \frac{\partial^{2} W}{\partial x^{2}}+p_{3} W+p_{4} \frac{\partial^{3} \Psi_{1}}{\partial x^{3}}+p_{5} \frac{\partial \Psi_{1}}{\partial x}+p_{6} \frac{\partial^{2} \Psi_{2}}{\partial x^{2}}+p_{7} \Psi_{2}=0 \\
& p_{8} \frac{\partial^{3} W}{\partial x^{3}}+p_{9} \frac{\partial W}{\partial x}+p_{10} \frac{\partial^{2} \Psi_{1}}{\partial x^{2}}+p_{11} \Psi_{1}+p_{12} \frac{\partial \Psi_{2}}{\partial x}=0 \\
& p_{13} \frac{\partial^{2} W}{\partial x^{2}}+p_{14} W+p_{15} \frac{\partial \Psi_{1}}{\partial x}+p_{16} \frac{\partial^{2} \Psi_{2}}{\partial x^{2}}+p_{17} \Psi_{2}=0
\end{aligned}
$$

where the coefficients of $p_{i}$ can be found in Appendix 5. It is worth noting that the coefficients $p_{2}$ and $p_{3}$ depend on temperature $(\Delta T)$ and moisture $(\Delta C)$. To solve the above partial differential equations in the $x$ direction, GDQ method is employed. According to this technique, the $r^{\text {th }}$-order partial derivative of a function $f(x)$ with respect to $x$ at a given point $x_{i}$ is estimated as follows:

$$
\frac{\partial^{r} f}{\partial x^{r}}=\sum_{k=1}^{N_{x}} c_{i k}^{r} f\left(x_{k}\right) \quad ; \quad i=, 2, \ldots, N_{x} \quad, \quad r=1,2, \ldots N_{x}-1
$$

where $c_{i k}^{r}$ are the weighting coefficients which can be found in Refs. [44, 45]. By applying GDQ approach into Eq. (26), the following discretized equations are obtained.

$$
\begin{aligned}
& \sum_{k=1}^{N_{x}}\left(p_{1} c_{i k}^{4}+p_{2} c_{i k}^{2}\right) W\left(x_{k}\right)+p_{3} W\left(x_{i}\right)+\sum_{k=1}^{N_{x}}\left(p_{4} c_{i k}^{3}+p_{5} c_{i k}^{1}\right) \Psi_{1}\left(x_{k}\right)+\sum_{k=1}^{N_{x}} p_{6} c_{i k}^{2} \Psi_{2}\left(x_{k}\right)+p_{7} \Psi_{2}\left(x_{i}\right)=0 \\
& \sum_{k=1}^{N_{x}}\left(p_{8} c_{i k}^{3}+p_{9} c_{i k}^{1}\right) W\left(x_{k}\right)+\sum_{k=1}^{N_{x}} p_{10} c_{i k}^{2} \Psi_{1}\left(x_{k}\right)+p_{11} \Psi_{1}\left(x_{i}\right)+\sum_{k=1}^{N_{x}} p_{12} c_{i k}^{1} \Psi_{2}\left(x_{k}\right)=0 \\
& \sum_{k=1}^{N_{x}} p_{13} c_{i k}^{2} W\left(x_{k}\right)+p_{14} W\left(x_{i}\right)+\sum_{k=1}^{N_{x}} p_{15} c_{i k}^{1} \Psi_{1}\left(x_{k}\right)+\sum_{k=1}^{N_{x}} p_{16} c_{i k}^{2} \Psi_{2}\left(x_{k}\right)+p_{17} \Psi_{2}\left(x_{i}\right)=0
\end{aligned}
$$

Next, after discretizing the partial differential buckling equations, the eigenvalue problem is obtained as:

$$
[\mathbf{S}]\left\{\begin{array}{l}
\left\{W_{d}\right\} \\
\left\{\Psi_{1 d}\right\} \\
\left\{\Psi_{2 d}\right\}
\end{array}\right\}=\{0\}
$$

in which $[\mathbf{S}]$ represents the stiffness matrix and $\left\{W_{d}\right\},\left\{\Psi_{1 d}\right\}$ and $\left\{\Psi_{2 d}\right\}$ are defined as:

$$
\left\{W_{d}\right\}=\left\{\begin{array}{l}
W_{2} \\
W_{3} \\
\ldots \\
W_{N_{x}-1}
\end{array}\right\} \quad, \quad\left\{\psi_{1 d}\right\}=\left\{\begin{array}{l}
\Psi_{1_{2}} \\
\Psi_{1_{3}} \\
\ldots \\
\Psi_{1_{N_{x}-1}}
\end{array}\right\} \quad, \quad\left\{\Psi_{2 d}\right\}=\left\{\begin{array}{l}
\Psi_{2_{2}} \\
\Psi_{2_{3}} \\
\ldots \\
\Psi_{2_{N_{x}-1}}
\end{array}\right\}
$$


It is crucial to note that, in general, $[\mathbf{S}]=\left[S_{d d}\right]-\left[S_{d b}\right]\left[S_{b b}\right]^{-1}\left[S_{b d}\right]$ which is obtained by discretizing both governing equations and boundary conditions. But, since the boundaries at edges $x=0, a$ are clamped, $\left[S_{b d}\right]$ becomes zero. Therefore, only Eq. (28) requires to be discretized and there is no need to discretize the boundary condition relations. In other words, $[\mathbf{S}]=\left[S_{d d}\right]$ which doesn't include the nodes on the boundaries. Now, the method of obtaining the critical buckling load is explained. To this end, at specific values of $n, \Delta T, \Delta C$, and $\lambda$, the amount of load $P_{x}$ is gradually increased until the determinant of the matrix $[S]$ becomes zero. Similar loads are calculated for different values of $n$, finally, their lowest value is considered as the critical buckling load of the structure.

\section{Fully clamped boundary conditions (CCCC)}

As the third type of boundary conditions, fully clamped sandwich plates are considered here. To obtain the critical buckling temperatures for this case, two-dimensional GDQ approach is implemented which approximates the partial derivatives of a continuous function $f(x, y)$ at point $(x, y)=\left(x_{i}, y_{j}\right)$ as:

$$
\begin{aligned}
& \left.\frac{\partial^{r} f}{\partial x^{r}}\right|_{\left(x_{i}, y_{j}\right)}=\sum_{k=1}^{N_{x}} c_{i k}^{r} f\left(x_{k}, y_{j}\right) \quad, \quad i=1, \ldots, N_{x}, j=1, \ldots, N_{y} \\
& \left.\frac{\partial^{s} f}{\partial y^{s}}\right|_{\left(x_{i}, y_{j}\right)}=\sum_{l=1}^{N_{y}} \bar{c}_{j l}^{s} f\left(x_{i}, y_{l}\right) \quad, \quad i=1, \ldots, N_{x}, j=1, \ldots, N_{y} \\
& \left.\frac{\partial^{(r+s)} f}{\partial x^{r} \partial y^{s}}\right|_{\left(x_{i}, y_{j}\right)}=\sum_{k=1}^{N_{x}} \sum_{l=1}^{N_{y}} c_{i k}^{r} \bar{c}_{j l}^{s} f\left(x_{k}, y_{l}\right) \quad, \quad i=1, \ldots, N_{x}, j=1, \ldots, N_{y}
\end{aligned}
$$

where $c_{i k}^{r}$ and $\bar{c}_{j l}^{s}$ are the weighting coefficients for $x$ and $y$ directions, respectively. By applying twodimensional GDQ method to Eq. (13), the following discretized equations are obtained.

$$
\begin{aligned}
& g_{11}^{(3)} \sum_{k=1}^{N_{x}} c_{i k}^{4} w\left(x_{k}, y_{j}\right)+\left(2 g_{12}^{(3)}+4 g_{66}^{(3)}\right) \sum_{k=1}^{N_{x}} \sum_{l=1}^{N_{y}} c_{i k}^{2} \bar{c}_{j l}^{2} w\left(x_{k}, y_{l}\right)+g_{22}^{(3)} \sum_{l=1}^{N_{y}} \bar{c}_{j l}^{4} w\left(x_{i}, y_{l}\right)+g_{11}^{(2)} \sum_{k=1}^{N_{x}} c_{i k}^{3} \psi_{1}\left(x_{k}, y_{j}\right)+ \\
& \left(g_{12}^{(2)}+2 g_{66}^{(2)}\right)\left(\sum_{k=1}^{N_{x}} \sum_{l=1}^{N_{y}} c_{i k}^{1} \bar{c}_{j l}^{2} \psi_{1}\left(x_{k}, y_{l}\right)+\sum_{k=1}^{N_{x}} \sum_{l=1}^{N_{y}} c_{i k}^{2} \bar{c}_{j l}^{1} \psi_{2}\left(x_{k}, y_{l}\right)\right)+g_{22}^{(2)} \sum_{l=1}^{N_{y}} \bar{c}_{j l}^{3} \psi_{2}\left(x_{i}, y_{l}\right)+ \\
& S_{c x z}\left(\sum_{k=1}^{N_{x}} c_{i k}^{1} \psi_{1}\left(x_{k}, y_{j}\right)-\sum_{k=1}^{N_{x}} c_{i k}^{2} w\left(x_{k}, y_{j}\right)\right)+S_{c y z}\left(\sum_{l=1}^{N_{y}} \bar{c}_{j l}^{1} \psi_{2}\left(x_{i}, y_{l}\right)-\sum_{l=1}^{N_{y}} \bar{c}_{j l}^{2} w\left(x_{i}, y_{l}\right)\right)+ \\
& \left(N_{x}^{T}+N_{x}^{M}+P_{x}\right) \sum_{k=1}^{N_{x}} c_{i k}^{2} w\left(x_{k}, y_{j}\right)+\left(N_{y}^{T}+N_{y}^{M}+P_{y}\right) \sum_{l=1}^{N_{y}} \bar{c}_{j l}^{2} w\left(x_{i}, y_{l}\right)=0
\end{aligned}
$$




$$
\begin{aligned}
& -g_{11}^{(2)} \sum_{k=1}^{N_{k}} c_{i k}^{3} w\left(x_{k}, y_{j}\right)-\left(g_{12}^{(2)}+2 g_{66}^{(2)}\right) \sum_{k=1}^{N_{x}} \sum_{l=1}^{N_{y}} c_{i k}^{1} \bar{c}_{j l}^{2} w\left(x_{k}, y_{l}\right)-\left(g_{12}^{(1)}+g_{66}^{(1)}\right) \sum_{k=1}^{N_{k}} \sum_{l=1}^{N_{y}} c_{i k}^{1} \bar{c}_{j l l}^{1} \psi_{2}\left(x_{k}, y_{l}\right) \\
& -g_{11}^{(1)} \sum_{k=1}^{N_{x}} c_{i k}^{2} \psi_{1}\left(x_{k}, y_{j}\right)-g_{66}^{(1)} \sum_{l=1}^{N_{y}} \bar{c}_{j l}^{2} \psi_{1}\left(x_{i}, y_{l}\right)+S_{c x z}\left(\psi_{1}\left(x_{i}, y_{j}\right)-\sum_{k=1}^{N_{x}} c_{i k}^{1} w\left(x_{k}, y_{j}\right)\right)=0 \\
& -g_{22}^{(2)} \sum_{l=1}^{N_{y}} \bar{c}_{j l}^{3} w\left(x_{i}, y_{l}\right)-\left(g_{12}^{(2)}+2 g_{66}^{(2)}\right) \sum_{i=1}^{N_{x}} \sum_{k=1}^{N_{y}} c_{i k}^{2} \bar{c}_{j l}^{1} w\left(x_{k}, y_{l}\right)-\left(g_{12}^{(1)}+g_{66}^{(1)}\right) \sum_{i=1}^{N_{k}} \sum_{k=1}^{N_{y}} c_{i k}^{1} \bar{c}_{j l l}^{1} \psi_{1}\left(x_{k}, y_{l}\right) \\
& -g_{22}^{(1)} \sum_{l=1}^{N_{y}} \bar{c}_{j l}^{2} \psi_{2}\left(x_{i}, y_{l}\right)-g_{66}^{(1)} \sum_{k=1}^{N_{k}} c_{i k}^{2} \psi_{2}\left(x_{k}, y_{j}\right)+S_{c y z}\left(\psi_{2}\left(x_{i}, y_{j}\right)-\sum_{l=1}^{N_{y}} \bar{c}_{j l}^{1} w\left(x_{i}, y_{l}\right)\right)=0
\end{aligned}
$$

The above equations can also be re-written in the following compact form.

$$
[\mathbf{S}]\left\{\begin{array}{l}
\left\{w_{d}\right\} \\
\left\{\psi_{1_{d}}\right\} \\
\left\{\psi_{2_{d}}\right\}
\end{array}\right\}=\{0\}
$$

where

$$
\begin{aligned}
& \left\{w_{d}\right\}=\left\{w_{2,2}, w_{2,3}, \ldots, w_{2, N_{y}-1}, \ldots, w_{N_{x}-1,2}, w_{N_{x}-1,3}, \ldots, w_{N_{x}-1, N_{y}-1}\right\}^{T} \\
& \left\{\psi_{1_{d}}\right\}=\left\{\psi_{1_{2,2}}, \psi_{1_{2,3}}, \ldots, \psi_{1_{2, N y}, 1}, \ldots, \psi_{1_{N_{x}-1,2}}, \psi_{1_{N_{x}-1,3}}, \ldots, \psi_{1_{N_{x}-1, N y-1}}\right\}^{T} \\
& \left\{\psi_{2_{d}}\right\}=\left\{\psi_{2_{2,2}}, \psi_{2_{2,3}}, \ldots, \psi_{2_{2, N_{y}-1}}, \ldots, \psi_{2_{N_{x}-1,2}}, \psi_{2_{N_{x}-1,3}}, \ldots, \psi_{2_{N_{x}-1, N,-1}}\right\}^{T}
\end{aligned}
$$

To obtain the critical buckling load, $P_{x}$ is gradually increased until the determinant of the matrix becomes zero. This load is reported as the critical buckling load.

\section{Results and discussion}

\subsection{Verification study}

In this subsection, the validity of present results is investigated. Five different comparisons are made here to show the accuracy of the results for a variety types of sandwich plates with different boundary conditions under various loadings. As the first example, mechanical buckling of a sandwich plate with soft core and isotropic skins under uniaxial and biaxial compressive loads is examined. The plate consists of two identical face sheets of thickness $0.5334 \mathrm{~mm}$ each and a core of $4.597 \mathrm{~mm}$ thick. The material properties of the sandwich plate in this example are as follows [46-48]:

$$
\begin{gathered}
\text { Core }: E_{x}=E_{y}=6.55 \times 10^{-3} \mathrm{~N} / \mathrm{mm}^{2} \quad ; \quad G_{x y}=0.262 \times 10^{-3} \mathrm{~N} / \mathrm{mm}^{2} \\
G_{x z}=G_{y z}=131.0 \mathrm{~N} / \mathrm{mm}^{2} \quad ; \quad v_{12}=0.25
\end{gathered}
$$

Face sheets $: E=65500.0 \mathrm{~N} / \mathrm{mm}^{2} \quad ; \quad v_{12}=0.25$ 
The comparison results are provided in Table 1 . In this table, critical buckling stress $\left(\sigma_{c r}=N_{x} / 2 h_{f}\right)$ of the sandwich plate which is exposed to uniaxial load $(\lambda=0)$ and biaxial loads $(\lambda=0.5)$ is evaluated for two types of boundary conditions and three different aspect ratios $(a / b)$ taking $b=596.9 \mathrm{~mm}$. From the comparison study of Table 1, two conclusions can be inferred. The first point is that the present results are in a very good agreement with other reported data in the literature [46-48]. The second point is about the convergency of the GDQ results. Numerical results of Table 1 for sandwich plates with fully clamped boundary conditions clarify that the applied GDQ method converges very fast and this method needs only $13 \times 13$ grid points to yield the converged responses.

The second example is related to the mechanical buckling of simply supported composite plates with soft core and two identical single-layer orthotropic skins $(\theta /$ Core $/ \theta)$. The structure is assumed to be under uniaxial compressive loading $(\lambda=0)$ and the results are tabulated for two different fiber orientations $\left(0^{\circ}\right.$ and $90^{\circ}$ ). The plate has a dimension of $225 \times 225 \mathrm{~mm}^{2}$ and the thickness of core and skins is $10 \mathrm{~mm}$ and $0.2 \mathrm{~mm}$, respectively. The martials properties are taken as follows [47, 49 and 50]:

Core $: E_{1}=E_{2}=0.02 G P a ; G_{12}=0.0146 G P a ; G_{13}=0.146 G P a$ $G_{23}=0.0904 G P a ; v_{12}=0.3$

Face sheets $: E_{1}=229 \mathrm{GPa} ; E_{2}=13.35 \mathrm{GPa} ; G_{12}=5.25 \mathrm{GPa} ; v_{12}=0.3151$

As Table 2 shows, the present results are very close to those available in Refs. [47, 49 and 50] and the differences between the results, which come from using various plate theories and solution methods, is little and ignorable.

In the next step, the present results are verified by evaluating thermal buckling results of rectangular sandwich plates. As the third example, a comparison is performed between the numerical results of present work and the results obtained by Shuia [28] and Ko [52] for critical buckling temperature of sandwich structures with aluminum honeycomb core and isotopic titanium face sheets (as shown in Fig. 2). The material properties of the core and skins for this example are assumed to be [52]:

$$
\begin{aligned}
\text { Honeycomb Core }: E_{x} & =2.7778 \times 10^{4} \mathrm{lb} / \mathrm{in}^{2} ; E_{y}=2.7778 \times 10^{4} \mathrm{lb} / \mathrm{in}^{2} ; E_{z}=2.7778 \times 10^{5} \mathrm{lb} / \mathrm{in}^{2} \\
G_{x y} & =0.00613 \times 10^{6} \mathrm{lb} / \mathrm{in}^{2} ; G_{x z}=1.81 \times 10^{5} \mathrm{lb} / \mathrm{in}^{2} ; G_{y z}=0.81967 \times 10^{5} \mathrm{lb} / \mathrm{in}^{2} \\
v_{x y} & =0.658 \times 10^{-2} ; \alpha_{x}=\alpha_{y}=5.37 \times 10^{-6} \mathrm{in} / \mathrm{in}-{ }^{o} \mathrm{~F}
\end{aligned}
$$

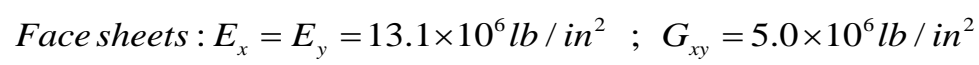

$$
v_{x y}=0.31 ; \alpha_{x}=\alpha_{y}=5.35 \times 10^{-6} \mathrm{in} / \mathrm{in}-{ }^{o} \mathrm{~F}
$$


The comparison shown in Table 3 represents that all three solution methods implemented in the present study can predict the critical buckling temperature of honeycomb sandwich plates very well for different boundary conditions. To verify the present results for thermal buckling of sandwich plates, one more comparison is here made. To this end, the buckling temperature of sandwich plates with soft core and two identical single-layer orthotropic skins $\left(0^{\circ} /\right.$ Core $\left./ 0^{\circ}\right)$ are evaluated. The structure is presumed to be exposed to environment with uninform temperature distribution. The material properties of the core and face sheets are considered as [35]:

$$
\begin{aligned}
& \text { Isotropic Core }: E=10 \mathrm{MPa} ; v_{12}=0.3 ; \alpha=0.18 \times 10^{-6} /{ }^{\circ} \mathrm{C} \\
& \text { Face sheets }: E_{1}=132 \mathrm{GPa} ; E_{2}=10.3 \mathrm{GPa} ; G_{12}=6.5 \mathrm{GPa} ; \\
& v_{12}=0.3 ; \alpha_{1}=1.2 \times 10^{-6} /{ }^{\circ} \mathrm{C} \quad ; \alpha_{2}=24 \times 10^{-6} /{ }^{\circ} \mathrm{C}
\end{aligned}
$$

Simply supported conditions are considered at all edges for this example. The comparison is performed for different $a / h$ ratios. It should be mentioned that the digitizer software with high accuracy was applied to obtain numerical data from the graphs of Ref. [35]. It can be concluded from Fig. 3 that the results obtained from the present research are so close to those reported by Zhai et al. [35] for thermal buckling analysis of sandwich plates.

Finally, a verification study is provided to compare the numerical results for hygrothermal buckling of a single-layer non-sandwich composite plate with thickness to length ratio of 0.01 . For this example, the material properties of the orthotropic layer are assumed as [22]:

$$
\begin{aligned}
& E_{1}=130 G P a ; E_{2}=9.5 G P a ; G_{12}=6 G P a ; v_{12}=0.3 \\
& \alpha_{1}=-0.3 \times 10^{-6} / K ; \alpha_{2}=28.1 \times 10^{-6} / K ; \beta_{1}=0 ; \beta_{2}=0.44
\end{aligned}
$$

In this case, the thickness of face sheets is set to zero $\left(h_{f}=0\right)$. It is important to note that since there is no face sheets here, a shear correction factor $\left(k_{s}\right)$ is required to zero the amount of stress at the top and bottom levels. The value of this factor is considered to be 5/6 in this example [22]. The comparison is shown in Table 4 for three different boundary conditions and two fiber orientations. It should be mentioned that, like the previous example, the digitizer software was employed to obtain the numerical results from the figures in Ref. [22]. As Table 4 represents, there is an excellent agreement between the present results and those reported by Ghadirian et al. [22].

Based on the above comparisons, it can be concluded that the results presented in this study are accurate enough and reliable for analyzing the buckling of rectangular sandwich plates with soft core and laminated face sheets in hygroscopic and thermal environments. 


\subsection{Numerical Results}

Now, buckling of soft-core sandwich plates with layered composite face sheets in hot and wet environments are studied. The results of this subsection are divided into two parts. As mentioned earlier, composite structures may buckle only due to heat expansion and moisture swelling without experiencing any mechanical pressure. Therefore, in the first part, the influences of operating temperature and humidity on the buckling of composite sandwich plates are examined when mechanical loads are not applied to the structure. Then, in the second part, the buckling of composite sandwich plates under hygrothermomechanical loads are discussed. In this case, the structure is under mechanical compressive loads which the critical values of these forces are evaluated for different hygroscopic and thermal conditions. Due to the limitation in the experimental data for cores, the properties of applied materials in the present work are considered to be temperature and moisture-independent, based on Refs. [53, 54], as follow:

$$
\begin{aligned}
& \text { Soft Core : } E=3 \mathrm{GPa} ; v=0.4 ; \alpha=50 \times 10^{-6} / \mathrm{K} ; \beta=0.28 \\
& \text { Face sheets : } E_{1}=138 \mathrm{GPa} ; E_{2}=8.5 \mathrm{GPa} ; G_{12}=G_{13}=4.5 \mathrm{GPa} ; G_{23}=3.2 \mathrm{GPa} \\
& \qquad v_{12}=0.29 ; \alpha_{1}=-0.5 \times 10^{-6} / \mathrm{K} ; \alpha_{2}=43 \times 10^{-6} / \mathrm{K} ; \beta_{1}=0 ; \beta_{2}=0.4
\end{aligned}
$$

Throughout this subsection, a three-layered composite with lamination scheme of $\left[0^{\circ} / 90^{\circ} / 0^{\circ}\right]$ is considered for the face sheets. Moreover, the thickness of each layers is assumed to $0.2 \mathrm{~mm}$ which means the face sheets are $0.6 \mathrm{~mm}$ thick.

\subsubsection{Hygrothermal buckling of composite sandwich plates (in the absence of in-plane mechanical forces)}

Fig. 4 illustrates the critical buckling temperatures and moisture contents for different core thickness values and three different types of boundary conditions. It is observed that in higher temperature environments, the critical moisture content which lead to buckling of the structure is lower. Similarly, this interpretation can be made that in environments with higher humidity, thermal buckling occurs at lower temperatures. Also, as expected, based on the hygrothermal loads term in Eq. (13) and considering that the properties of the materials do not depend on temperature and moisture, it is concluded that the graphs shown in Fig. 4 are changed linearly. 
One of the important issues in designing of sandwich structures is the estimation of the required core thickness. Therefore, in Fig. 5, the minimum core thickness required to avoid hygrothermal buckling in the sandwich structure is shown for different environments. As this figure elucidates, with the increase of temperature or humidity, the core of sandwich plate needs to be thicker. This is because with increasing the temperature or the moisture of the environment, the in-plane compressive loads increase which lead to a decrease in total stiffness of the structure. Thus, more thickness is needed to enhance the stiffness of structure and delay buckling phenomenon in the structure. In Fig. 8 the variations of critical buckling temperature as well as critical buckling moisture content are shown versus aspect ratio parameter $(b / a)$. The structure is assumed to be simply supported at all the edges and the aspect ratio parameter varies from 1 to 5. As the figure illustrates, there is a decreasing-increasing trend for the changes of $\Delta T_{c r}$ and $\Delta M_{c r}$. This is because the critical buckling temperature and critical buckling moisture content, depending on the aspect ratio value, may occur at different values of $m$ and $n$, based on Eqs. (21-24). It can be also concluded that only $25^{\circ} \mathrm{C}$ increase in temperature or $0.1 \%$ moisture in the structure can affectively decrease the buckling limits.

\subsubsection{Effects of hygrothermal environments on the buckling of composite sandwich plates under uniaxial/biaxial mechanical compressive loads}

Consider the sandwich plate of Fig. 1 in which the structure is under uniaxial compressive load ( $\lambda=0$ ). For this case, as the humidity and temperature status changes, the critical load changes. Fig. 7 depicts the variations of buckling load of simply supported sandwich plates for various hygroscopic and thermal conditions. The figure illustrates that with the increase of temperature or moisture content, the critical buckling load of the structure decreases. Similar to what mentioned before for Fig. 4, the variations of buckling load against both temperature or moisture is expected to be linear. Flaggs and Vinson [17] obtained almost similar conclusion for buckling analysis of non-sandwich composite plates under hygrothermal effects. From hygroscopic point of view, they assumed that the material properties are moisture-independent. But, considering the effect of temperature on the material features, they measured the material properties of the structure at two different temperature, $70^{\circ} \mathrm{F}$ and $350^{\circ} \mathrm{F}$. Then, a linear variation was presumed for the properties between these two temperatures. As a result, it was shown that critical buckling linearly reduces with increasing moisture content or temperatures (if temperature is in a low range) but nonlinearly decreases at high temperatures (especially for $T>250^{\circ} \mathrm{F}$ ). Similarly, Ram and 
Sinha [19] concluded the same results for variations of buckling load versus moisture content or temperature.

Figures 9 and 9 show the role of core thickness in buckling of simply supported rectangular sandwich plates for a variety hygrothermal conditions. In Fig. 8, some comparisons are made for the response of the structure in buckling between when the structure is in the environment at room temperature without humidity, and when the structure is applied in hygroscopic and thermal conditions. In this figure, the variations of critical buckling load reduction rate $\left(100 \times \frac{N_{c r}-N_{c r}^{0}}{N_{c r}^{0}}\right)$ are shown for different operating temperatures and moisture contents and various core thicknesses. $N_{c r}^{0}$ denotes the critical buckling load of the structure in an environment with room temperature and without moisture. As expected, the hygrothermal environments play an important role in buckling of sandwich plates, especially when their cores are thinner. It is found that as the core thickness increases, the effect of moisture and temperature on critical buckling of the structure significantly decreases. By focusing on Fig. 9, it is inferred that the core thickness of sandwich structures considerably affects the performance of sandwich plates in buckling. For example, at conditions in which $\Delta T=0$ and $\Delta C=1.5 \%$, when the core is $11.125 \mathrm{~mm}$ thick, the sandwich plate cannot withstand any compressive load. But, if the thickness of core is $20 \mathrm{~mm}$, the structure can withstand about $977 \mathrm{KN} / \mathrm{m}$ uniaxial load. From Fig. 9, it is also seen that with the increase of core thickness, the critical buckling load non-linearly increase at different hygrothermal environments. For further investigation regarding the influence of core thickness on the behavior of sandwich plates, some numerical results are provided in Tables 5 and 6. In these tables, critical uniaxial buckling loads are calculated for different environments and three types of boundary conditions.

The effect of length to thickness parameter on buckling load of a simply supported square sandwich plate is investigated in Fig. 10. As it is observed, when the sandwich plate thins ( $a / h$ ratio increases), its buckling load decreases. The graphs of Fig. 10 elucidate that hygroscopic and thermal conditions seriously affect the response of structure in buckling. It is worth noting that the $a / h$ ratio for which the buckling load becomes zero is the minimum length to thickness ratio which prevent buckling phenomenon in the structure.

Finally, the influence of aspect ratio parameter on the buckling of composite sandwich plates are depicted in Figs. 11 and 12 for various hygrothermal conditions and mechanical loads. In Fig. 11, it is assumed that a uniaxial load is applied on the structure. This figure indicates that as the plate gets longer, the critical buckling load continuously reduces. This figure also represents that all critical buckling loads occur at $n=1$ , $m=1$. Furthermore, it can be found that moisture content and temperature have an effective role in stability of sandwich plates. Another kind of loading is considered in Fig. 12 for soft-core sandwich plate 
exposed to hygrothermal environment. In this case, the structure is supposed to be under biaxial compressive loads with $\lambda=1$. Like Fig. 6 , there is a decreasing-increasing trend for variations of buckling load against $b / a$ parameter. The reason is that thermal buckling occurs at different half-wave numbers, depending on the geometry of the structure, as discussed earlier.

\section{Conclusion}

In this research work, the buckling of sandwich plates with layered composite face sheets under hygrothermal environments was studied. The stability equations and related boundary conditions were derived based on a piecewise shear deformations theory. Depending on the type of boundary conditions, three analytical, semi-analytical and numerical methods were applied for the analysis. First, the accuracy of present results was shown by performing a comprehensive verification study. Then, a parametric study was carried out to indicate the effects of hot and wet conditions on the buckling of sandwich plates for different geometrical parameters and boundary conditions. From the present study, some conclusions can be drawn:

- PLSDT can appropriately provide stability equations and boundary conditions for the buckling analysis of sandwich structures subjected to hygro-thermo-mechanical loadings.

- Using PLSDT for analysis of sandwich plates creates the difficulty that the number of boundary conditions and the number of stability equations are not the same. The present study shows that this problem can be successfully overcome by using an analytical solution for simply supported boundaries and GDQ method for clamped edges.

- The numerical comparisons clarify that the applied methods in the present work can predict the buckling of sandwich structures subjected hygroscopic and thermal environments with high accuracy.

- Results show that wet and hot environments significantly affect the buckling of sandwich plates, especially when their cores are thin.

\section{Acknowledgement}

This work was supported by the National Research Foundation of Korea (NRF) grant funded by Korea government (2018R1A6A1A03024509, 2019R1A2B5B03004980). 


\section{References}

[1] Adim, Belkacem, Tahar Hassaine Daouadji, Boussad Abbes, and A. Rabahi. "Buckling and free vibration analysis of laminated composite plates using an efficient and simple higher order shear deformation theory." Mechanics \& Industry 17, no. 5 (2016).

[2] Abdelhak, Z., L. Hadji, Z. Khelifa, T. Hassaine Daouadji, and E. A. Adda Bedia. "Analysis of buckling response of functionally graded sandwich plates using a refined shear deformation theory." Wind and Structures 22, no. 3 (2016): 291-305.

[3] Jelovica, J., and J. Romanoff. "Buckling of sandwich panels with transversely flexible core: Correction of the equivalent single-layer model using thick-faces effect." Journal of Sandwich Structures \& Materials (2018): 1099636218789604.

[4] Belkacem, Adim, Hassaine Daouadji Tahar, Rabahi Abderrezak, Benhenni Mohamed Amine, Zidour Mohamed, and Abbes Boussad. "Mechanical buckling analysis of hybrid laminated composite plates under different boundary conditions." Structural Engineering and Mechanics 66, no. 6 (2018): 761-769.

[5] Daouadji, Tahar Hassaine, and Belkacem Adim. "An analytical approach for buckling of functionally graded plates." Advances in materials Research 5, no. 3 (2016): 141.

[6] Amir, Saeed, Mohammad Khorasani, and Hassan BabaAkbar-Zarei. "Buckling analysis of nanocomposite sandwich plates with piezoelectric face sheets based on flexoelectricity and first-order shear deformation theory." Journal of Sandwich Structures \& Materials (2018): 1099636218795385.

[7] Moradi-Dastjerdi, Rasool, and Kamran Behdinan. "Stability analysis of multifunctional smart sandwich plates with graphene nanocomposite and porous layers." International Journal of Mechanical Sciences 167 (2020): 105283.

[8] Safaei, Babak, Rasool Moradi-Dastjerdi, Kamran Behdinan, and Fulei Chu. "Critical buckling temperature and force in porous sandwich plates with CNT-reinforced nanocomposite layers." Aerospace Science and Technology 91 (2019): 175-185.

[9] Abdelhak, Zohra, Lazreg Hadji, T. Hassaine Daouadji, and E. A. Adda Bedia. "Thermal buckling response of functionally graded sandwich plates with clamped boundary conditions." Smart Structures and Systems 18, no. 2 (2016): 267-291.

[10] Atteshamuddin S Sayyad, and Y. M Ghugal. "On the buckling of advanced composite sandwich rectangular plates Journal of Sandwich Structures \& Materials (2020), Published Online. https://doi.org/10.1177/1099636220925084.

[11] Kaw, Autar K. Mechanics of composite materials. CRC press, 2005.

[12] Alam, Parvez, Colin Robert, and Conchúr M. Ó. Brádaigh. "Tidal turbine blade composites-A review on the effects of hygrothermal aging on the properties of CFRP." Composites Part B: Engineering 149 (2018): 248-259.

[13] Ahmad, Muhammad Riaz, Bing Chen, M. Aminul Haque, and Syed Farasat Ali Shah. "Development of a sustainable and innovant hygrothermal bio-composite featuring the enhanced mechanical properties." Journal of Cleaner Production 229 (2019): 128-143.

[14] Rahnama, Saeed, Roham Rafiee, and Mehdi Maleki. "The influence of hygrothermal environments on the stress concentration in unidirectional composite lamina." Mechanics of Materials 129 (2019): 332-340.

[15] Avva, V. Sarma. Buckling of Composites Under Hot and Wet Environment. Vol. 84, no. 3071. Flight Dynamics Laboratory, Air Force Wright Aeronautical Laboratories, Air Force Systems Command, 1984.

[16] Whitney, J. M., and J. E. Ashton. "Effect of environment on the elastic response of layered composite plates." AIAA Journal 9, no. 9 (1971): 1708-1713. 
[17] Flaggs, Donald L., and Jack R. Vinson. "Hydrothermal effects on the buckling of laminated composite plates." Fibre Science and Technology 11, no. 5 (1978): 353-365.

[18] Ram, KS Sai, and P. K. Sinha. "Vibration and buckling of laminated plates with a cutout in hygrothermal environment." AIAA journal 30, no. 9 (1992): 2353-2355.

[19] Ram, KS Sai, and P. K. Sinha. "Hygrothermal effects on the buckling of laminated composite plates." Composite Structures 21, no. 4 (1992): 233-247.

[20] Chao, Lu-Ping, and Shyh-Long Shyu. "Nonlinear buckling of fiber-reinforced composite plates under hygrothermal effects." Journal of the Chinese Institute of Engineers 19, no. 6 (1996): 657-667.

[21] Sreehari, V. M., and D. K. Maiti. "Buckling and post buckling analysis of laminated composite plates in hygrothermal environment using an Inverse Hyperbolic Shear Deformation Theory." Composite Structures 129 (2015): 250-255.

[22] Ghadirian, H., MR Ghazavi, and K. Khorshidi. "Vibration and stability analysis of laminated composite plates with hygrothermal effects." (2016): 155-166.

[13] Sreehari, V. M., and D. K. Maiti. "Buckling load enhancement of damaged composite plates under hygrothermal environment using unified particle swarm optimization." Structural and Multidisciplinary Optimization 55, no. 2 (2017): 437-447.

[24] Chen, C. S., T. C. Tsai, T. J. Chen, and W. R. Chen. "Vibration and Stability of Initially Stressed Hybrid Composite Plates in Hygrothermal Environments." Mechanics of Composite Materials 53, no. 4 (2017): 441-456.

[25] Amoushahi, Hossein, and Farshad Goodarzian. "Dynamic and buckling analysis of composite laminated plates with and without strip delamination under hygrothermal effects using finite strip method." Thin-Walled Structures 131 (2018): 88-101.

[26] Babu, C. Sarath, and T. Kant. "Refined higher order finite element models for thermal buckling of laminated composite and sandwich plates." Journal of thermal stresses 23, no. 2 (2000): 111-130.

[27] Kant, T. A. R. U. N., and C. S. Babu. "Thermal buckling analysis of skew fibre-reinforced composite and sandwich plates using shear deformable finite element models." Composite Structures 49, no. 1 (2000): 77-85.

[28] Shiau, Le-Chung, and Shih-Yao Kuo. "Thermal buckling of composite sandwich plates." Mechanics based design of structures and machines 32, no. 1 (2004): 57-72.

[29] Matsunaga, Hiroyuki. "Thermal buckling of cross-ply laminated composite and sandwich plates according to a global higher-order deformation theory." Composite Structures 68, no. 4 (2005): 439-454.

[30] Matsunaga, Hiroyuki. "Thermal buckling of cross-ply laminated composite and sandwich plates according to a global higher-order deformation theory." Composite Structures 68, no. 4 (2005): 439-454.

[31] Vangipuram, Pradeep, and N. Ganesan. "Buckling and vibration of rectangular composite viscoelastic sandwich plates under thermal loads." Composite Structures 77, no. 4 (2007): 419-429.

[32] Pradeep, V., and N. Ganesan. "Thermal buckling and vibration behavior of multi-layer rectangular viscoelastic sandwich plates." Journal of Sound and Vibration 310, no. 1-2 (2008): 169-183.

[33] Han, Bin, Ke-Ke Qin, Qian-Cheng Zhang, Qi Zhang, Tian Jian Lu, and Bing-Heng Lu. "Free vibration and buckling of foam-filled composite corrugated sandwich plates under thermal loading." Composite Structures 172 (2017): 173-189.

[34] Vescovini, Riccardo, M. d'Ottavio, Lorenzo Dozio, and O. Polit. "Thermal buckling response of laminated and sandwich plates using refined 2-D models." Composite Structures 176 (2017): 313-328.

[35] Zhai, Yanchun, Jianmin Su, and Sen Liang. "Free vibration and buckling analysis of composite sandwich plates in thermal environment." Journal of Sandwich Structures \& Materials (2018): 1099636218795375. 
[36] Birman, V. and Kardomateas, G.A., 2018. Review of current trends in research and applications of sandwich structures. Composites Part B: Engineering, 142, pp.221-240.

[37] Khan, Tayyab, Volkan Acar, M. Raci Aydin, Burak Hülagü, Hamit Akbulut, and M. Özgür Seydibeyoğlu. "A review on recent advances in sandwich structures based on polyurethane foam cores." Polymer Composites.

[38] Li, Xiangyang, Kaiping Yu, Jingyong Han, Rui Zhao, and Ying Wu. "A piecewise shear deformation theory for free vibration of composite and sandwich panels." Composite Structures 124 (2015): 111-119.

[39] Taheri, F., and S. Moradi. "A robust methodology for the simulation of postbuckling response of composite plates." Computational Mechanics 26, no. 3 (2000): 295-301.

[40] Kamarian, S., and M. Shakeri. "Thermal buckling analysis and stacking sequence optimization of rectangular and skew shape memory alloy hybrid composite plates." Composites Part B: Engineering 116 (2017): 137-152.

[41] Ghommem, M., and A. Abdelkefi. "Nonlinear reduced-order modeling and effectiveness of electrically-actuated microbeams for bio-mass sensing applications." International Journal of Mechanics and Materials in Design 15, no. 1 (2019): 125-143.

[42] Kamarian, S., M. Shakeri, and M. H. Yas. "Natural frequency analysis and optimal design of CNT/fiber/polymer hybrid composites plates using mori-tanaka approach, GDQ technique, and firefly algorithm." Polymer Composites 39, no. 5 (2018): 1433-1446.

[43] Reddy, Junuthula Narasimha. Mechanics of laminated composite plates and shells: theory and analysis. CRC press, 2003.

[44] Shu, Chang, and Bryan E. Richards. "Application of generalized differential quadrature to solve two-dimensional incompressible Navier-Stokes equations." International Journal for Numerical Methods in Fluids 15, no. 7 (1992): 791-798.

[45] Tornabene, Francesco, Nicholas Fantuzzi, Francesco Ubertini, and Erasmo Viola. "Strong formulation finite element method based on differential quadrature: a survey." Applied Mechanics Reviews 67, no. 2 (2015).

[46] Khatua, T. P., and Y. K. Cheung. "Stability analysis of multilayer sandwich structures." AIAA Journal 11, no. 9 (1973): 1233-1234.

[47] Yuan, W. X., and D. J. Dawe. "Overall and local buckling of sandwich plates with laminated faceplates, part II: Applications." Computer methods in applied mechanics and engineering 190, no. 40-41 (2001): 5215-5231.

[48] Pandit, M. K., B. N. Singh, and A. H. Sheikh. "Buckling of laminated sandwich plates with soft core based on an improved higher order zigzag theory." Thin-Walled Structures 46, no. 11 (2008): 1183-1191.

[49] Rao KM. Buckling analysis of anisotropic sandwich plates faced with fiber reinforced plastics. AIAA J 1985;23(8):1247-53.

[50] Kim, Chun-Gon, and Chang Sun Hong. "Buckling of unbalanced anisotropic sandwich plates with finite bonding stiffness." AIAA journal 26, no. 8 (1988): 982-988.

[51] Shariyat, M. "Non-linear dynamic thermo-mechanical buckling analysis of the imperfect sandwich plates based on a generalized three-dimensional high-order global-local plate theory." Composite Structures 92, no. 1 (2010): 72 85 .

[52] Ko, William L. "Predictions of thermal buckling strengths of hypersonic aircraft sandwich panels using minimum potential energy and finite element methods." (1995).

[53] Brischetto, Salvatore. "Hygrothermoelastic analysis of multilayered composite and sandwich shells." Journal of Sandwich Structures \& Materials 15, no. 2 (2013): 168-202.

[54] Ahmadi, Isa. "Edge stresses analysis in laminated thick sandwich cylinder subjected to distributed hygrothermal loading." Journal of Sandwich Structures \& Materials 20, no. 4 (2018): 425-461. 


\section{Appendix 1}

$\bar{Q}_{11}=Q_{11} \cos ^{4} \theta+2\left(Q_{12}+2 Q_{66}\right) \operatorname{Sin}^{2} \theta \operatorname{Cos}^{2} \theta+Q_{22} \operatorname{Sin}^{4} \theta$

$\bar{Q}_{12}=\left(Q_{11}+Q_{22}-4 Q_{66}\right) \operatorname{Sin}^{2} \theta \operatorname{Cos}^{2} \theta+Q_{12}\left(\operatorname{Sin}^{4} \theta+\operatorname{Cos}^{4} \theta\right)$

$\bar{Q}_{22}=Q_{11} \operatorname{Sin}^{4} \theta+2\left(Q_{12}+2 Q_{66}\right) \operatorname{Sin}^{2} \theta \operatorname{Cos}^{2} \theta+Q_{22} \operatorname{Cos}^{4} \theta$

$\bar{Q}_{16}=\left(Q_{11}-Q_{12}-2 Q_{66}\right) \operatorname{Sin} \theta \operatorname{Cos}^{3} \theta+\left(Q_{12}-Q_{22}+2 Q_{66}\right) \operatorname{Sin}^{3} \theta \operatorname{Cos} \theta$

$\bar{Q}_{26}=\left(Q_{11}-Q_{12}-2 Q_{66}\right) \operatorname{Sin}^{3} \theta \operatorname{Cos} \theta+\left(Q_{12}-Q_{22}+2 Q_{66}\right) \operatorname{Sin} \theta \operatorname{Cos}^{3} \theta$

$\bar{Q}_{66}=\left(Q_{11}+Q_{22}-2 Q_{12}-2 Q_{66}\right) \operatorname{Sin}^{2} \theta \operatorname{Cos}^{2} \theta+Q_{66}\left(\operatorname{Sin}^{4} \theta+\operatorname{Cos}^{4} \theta\right)$

$\bar{Q}_{44}=Q_{44} \operatorname{Cos}^{2} \theta+Q_{55} \operatorname{Sin}^{2} \theta$

$\bar{Q}_{45}=\left(Q_{55}-Q_{44}\right) \operatorname{Cos} \theta \operatorname{Sin} \theta$

$\bar{Q}_{55}=Q_{44} \operatorname{Sin}^{2} \theta+Q_{55} \operatorname{Cos}^{2} \theta$

where $\theta$ is the angle that the fibers of $k^{\text {th }}$ lamina makes with the structure x-axis, and $Q_{i j}$, called the plane stress-reduced stiffnesses, are given by:

$$
\begin{aligned}
Q_{11} & =\frac{E_{1}}{1-v_{12} v_{21}} \\
Q_{12} & =\frac{v_{12} E_{2}}{1-v_{12} v_{21}}=\frac{v_{21} E_{1}}{1-v_{12} v_{21}} \\
Q_{22} & =\frac{E_{2}}{1-v_{12} v_{21}} \\
Q_{44} & =G_{23} \\
Q_{55} & =G_{13} \\
Q_{66} & =G_{12}
\end{aligned}
$$

Where, $E_{1}, E_{2}, v_{12}$ and $G_{12}$ are the independent material constants [43]. 


\section{Appendix 2}

Thermal expansion coefficients [43]:

$\alpha_{x}=\alpha_{1} \cos ^{2} \theta+\alpha_{2} \sin ^{2} \theta$

$\alpha_{y}=\alpha_{1} \sin ^{2} \theta+\alpha_{2} \cos ^{2} \theta$

$\alpha_{x y}=2\left(\alpha_{1}-\alpha_{2}\right) \operatorname{Sin} \theta \cos \theta$

where $\alpha_{1}$ and $\alpha_{2}$ are the longitudinal and transverse coefficients of thermal expansion of the composite material.

Moist expansion coefficients[43]:

$\beta_{x}=\beta_{1} \cos ^{2} \theta+\beta_{2} \sin ^{2} \theta$

$\beta_{y}=\beta_{1} \sin ^{2} \theta+\beta_{2} \cos ^{2} \theta$

$\beta_{x y}=2\left(\beta_{1}-\beta_{2}\right) \operatorname{Sin} \theta \cos \theta$

where $\beta_{1}$ and $\beta_{2}$ are the longitudinal and transverse coefficients of moisture expansion of the composite material. 


\section{Appendix 3}

$$
\begin{aligned}
& g_{11}^{(1)}=2\left(\frac{2 h_{c}+h_{f}}{2}\right)^{2} A_{11}^{f}+\left(\frac{2 h_{c}+h_{f}}{2 h_{c}}\right)^{2} D_{11}^{c} \\
& g_{22}^{(1)}=2\left(\frac{2 h_{c}+h_{f}}{2}\right)^{2} A_{22}^{f}+\left(\frac{2 h_{c}+h_{f}}{2 h_{c}}\right)^{2} D_{22}^{c} \\
& g_{12}^{(1)}=2\left(\frac{2 h_{c}+h_{f}}{2}\right)^{2} A_{12}^{f}+\left(\frac{2 h_{c}+h_{f}}{2 h_{c}}\right)^{2} D_{12}^{c} \\
& g_{66}^{(1)}=2\left(\frac{2 h_{c}+h_{f}}{2}\right)^{2} A_{66}^{f}+\left(\frac{2 h_{c}+h_{f}}{2 h_{c}}\right)^{2} D_{66}^{c} \\
& g_{11}^{(2)}=\left(\frac{h_{f}}{2 h_{c}}\right)\left(\frac{2 h_{c}+h_{f}}{2 h_{c}}\right) D_{11}^{c} \\
& g_{22}^{(2)}=\left(\frac{h_{f}}{2 h_{c}}\right)\left(\frac{2 h_{c}+h_{f}}{2 h_{c}}\right) D_{22}^{c} \\
& g_{12}^{(2)}=\left(\frac{h_{f}}{2 h_{c}}\right)\left(\frac{2 h_{c}+h_{f}}{2 h_{c}}\right) D_{12}^{c} \\
& g_{66}^{(2)}=\left(\frac{h_{f}}{2 h_{c}}\right)\left(\frac{2 h_{c}+h_{f}}{2 h_{c}}\right) D_{66}^{c} \\
& g_{11}^{(3)}=2 D_{11}^{f}+\left(\frac{h_{f}}{2 h_{c}}\right)^{2} D_{11}^{c} \\
& g_{22}^{(3)}=2 D_{22}^{f}+\left(\frac{h_{f}}{2 h_{c}}\right)^{2} D_{22}^{c} \\
& g_{12}^{(3)}=2 D_{12}^{f}+\left(\frac{h_{f}}{2 h_{c}}\right)^{2} D_{12}^{c} \\
& g_{66}^{(3)}=2 D_{66}^{f}+\left(\frac{h_{f}}{2 h_{c}}\right)^{2} D_{66}^{c}
\end{aligned}
$$




\section{Appendix 4}

$$
\begin{aligned}
& k_{11}=\left(\frac{m \pi}{a}\right)^{4} g_{11}^{(3)}+\left(\frac{m \pi}{a}\right)^{2}\left(\frac{n \pi}{b}\right)^{2}\left(2 g_{12}^{(3)}+4 g_{66}^{(3)}\right)+\left(\frac{n \pi}{b}\right)^{4} g_{22}^{(3)}+\left(\frac{m \pi}{a}\right)^{2}\left(S_{c x z}-N_{x}^{T}-N_{x}^{H}-P_{x}\right)+\left(\frac{n \pi}{b}\right)^{2}\left(S_{c y z}-N_{y}^{T}-N_{y}^{H}-P_{y}\right) \\
& k_{12}=\left(\frac{m \pi}{a}\right)^{3} g_{11}^{(2)}+\left(\frac{m \pi}{a}\right)\left(\frac{n \pi}{b}\right)^{2}\left(g_{12}^{(2)}+2 g_{66}^{(2)}\right)-\left(\frac{m \pi}{a}\right) S_{c x z} \\
& k_{13}=\left(\frac{n \pi}{b}\right)^{3} g_{22}^{(2)}+\left(\frac{m \pi}{a}\right)^{2}\left(\frac{n \pi}{b}\right)\left(g_{12}^{(2)}+2 g_{66}^{(2)}\right)-\left(\frac{n \pi}{b}\right) S_{c y z} \\
& k_{21}=k_{12} \\
& k_{22}=\left(\frac{m \pi}{a}\right)^{2} g_{11}^{(1)}+\left(\frac{n \pi}{b}\right)^{2} g_{66}^{(1)}+S_{c x z} \\
& k_{23}=\left(\frac{m \pi}{a}\right)\left(\frac{n \pi}{b}\right)\left(g_{12}^{(1)}+g_{66}^{(1)}\right) \\
& k_{31}=k_{13} \\
& k_{32}=k_{23} \\
& k_{33}=\left(\frac{n \pi}{b}\right)^{2} g_{22}^{(1)}+\left(\frac{m \pi}{a}\right)^{2} g_{66}^{(1)}+S_{c y z}
\end{aligned}
$$




\section{Appendix 5}

$$
\begin{aligned}
& p_{1}=g_{11}^{(3)} \\
& p_{2}=-\left(\frac{n \pi}{b}\right)^{2}\left(2 g_{12}^{(3)}+4 g_{66}^{(3)}\right)+\left(N_{x}^{T}+N_{x}^{H}+P_{x}-S_{c x z}\right) \\
& P_{3}=\left(\frac{n \pi}{b}\right)^{4} g_{22}^{(3)}+\left(\frac{n \pi}{b}\right)^{2}\left(S_{c y z}-N_{y}^{T}-N_{y}^{H}-P_{y}\right) \\
& P_{4}=g_{11}^{(2)} \\
& P_{5}=-\left(\frac{n \pi}{b}\right)^{2}\left(g_{12}^{(2)}+2 g_{66}^{(2)}\right)+S_{c x z} \\
& P_{6}=-\left(\frac{n \pi}{b}\right)\left(g_{12}^{(2)}+2 g_{66}^{(2)}\right) \\
& p_{7}=\left(\frac{n \pi}{b}\right)^{3} g_{22}^{(2)}-\left(\frac{n \pi}{b}\right) S_{c y z} \\
& p_{8}=-g_{11}^{(2)} \\
& p_{9}=\left(\frac{n \pi}{b}\right)^{2}\left(g_{12}^{(2)}+2 g_{66}^{(2)}\right)-S_{c x z} \\
& p_{10}=-g_{11}^{(1)} \\
& p_{11}=\left(\frac{n \pi}{b}\right)^{2} g_{66}^{(1)}+S_{c x z} \\
& p_{12}=\left(\frac{n \pi}{b}\right)\left(g_{12}^{(1)}+g_{66}^{(1)}\right) \\
& p_{13}=-\left(\frac{n \pi}{b}\right)\left(g_{12}^{(2)}+2 g_{66}^{(2)}\right) \\
& p_{14}=\left(\frac{n \pi}{b}\right)^{3} g_{22}^{(2)}-\left(\frac{n \pi}{b}\right) S_{c y z} \\
& p_{15}=-\left(\frac{n \pi}{b}\right)\left(g_{12}^{(1)}+g_{66}^{(1)}\right) \\
& p_{16}=-g_{66}^{(1)} \\
& p_{17}=\left(\frac{n \pi}{b}\right)^{2} g_{22}^{(1)}+S_{c y z} \\
& p^{(1)}
\end{aligned}
$$



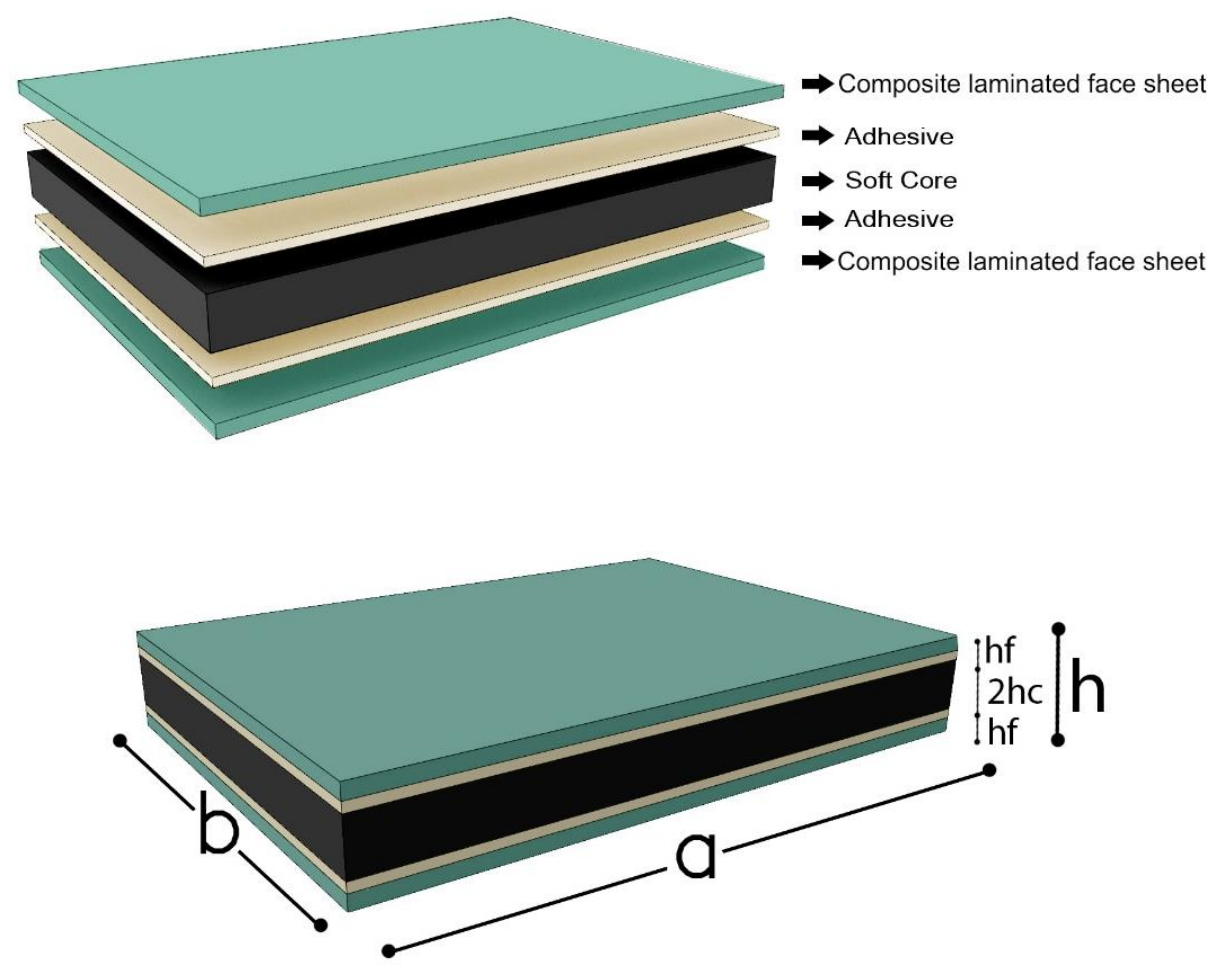

Fig 1 Schematic of a rectangular soft-core sandwich plate with layered composite face sheets 

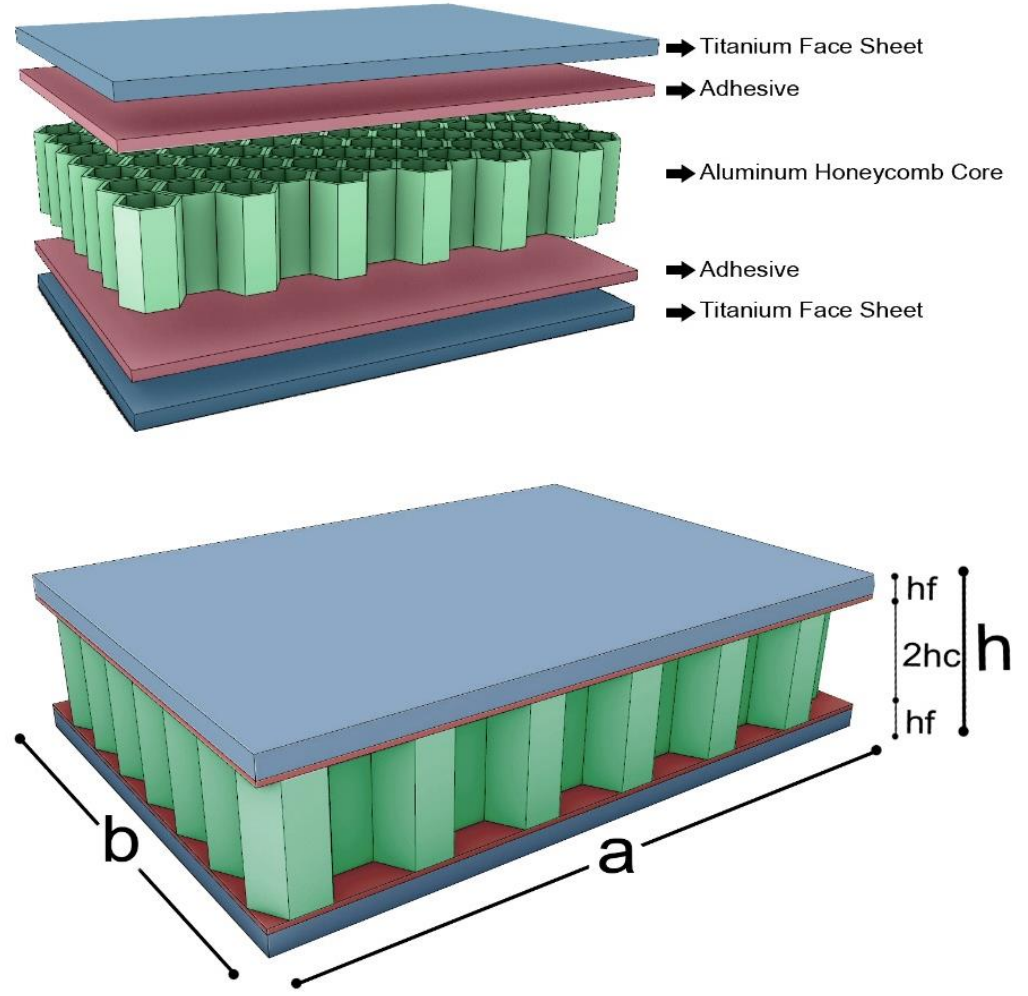

Fig 2 Schematic of a rectangular sandwich plate with aluminum honeycomb core and titanium face sheets 


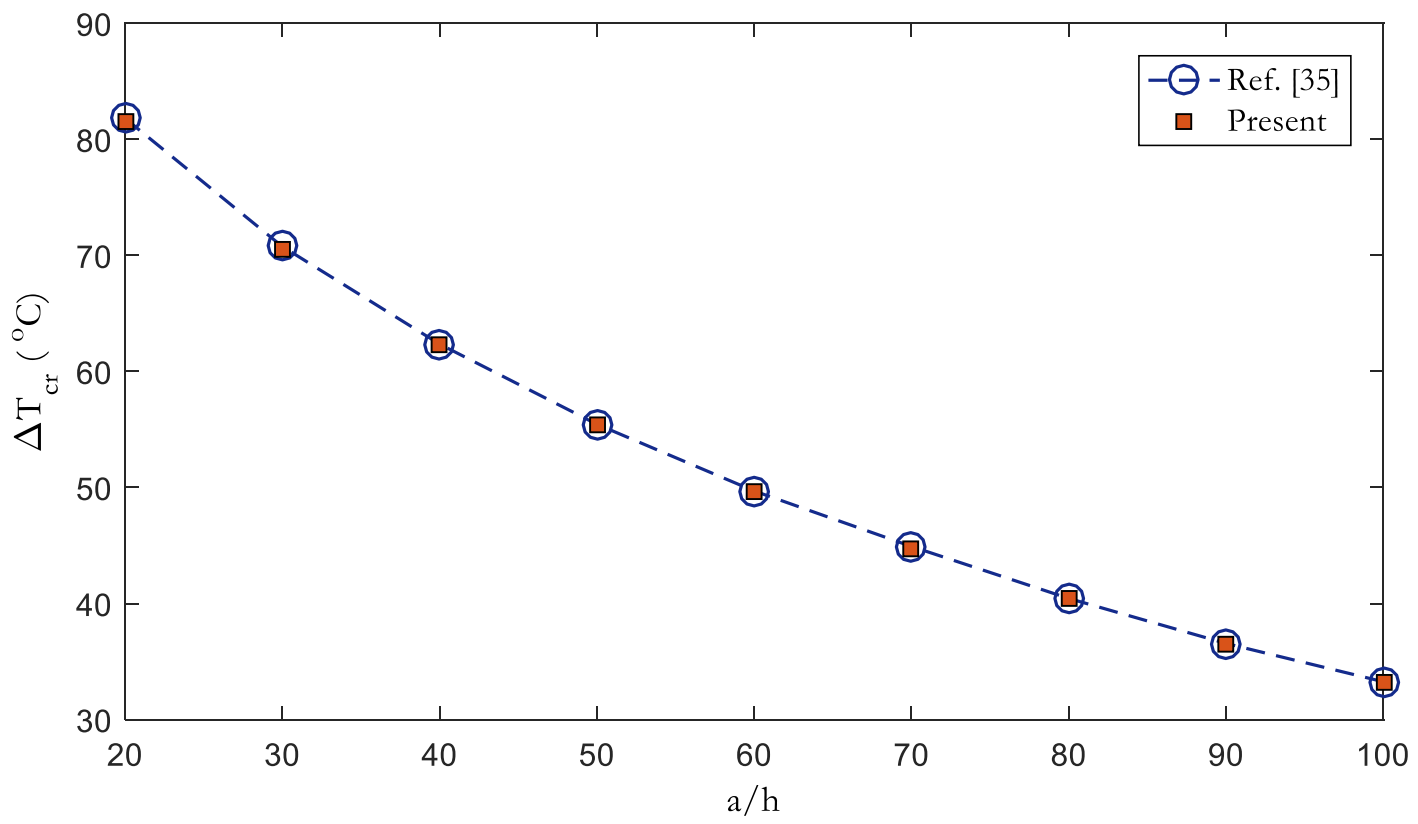

Fig 3 Comparison of the present results for critical buckling temperature prediction of a simply supported sandwich structure with soft core and single-layer orthotropic skins

$$
\left(a=0.4 \mathrm{~m}, b=0.3 \mathrm{~m}, h=10 \mathrm{~mm}, h_{f}=0.5 \mathrm{~mm}\right)
$$



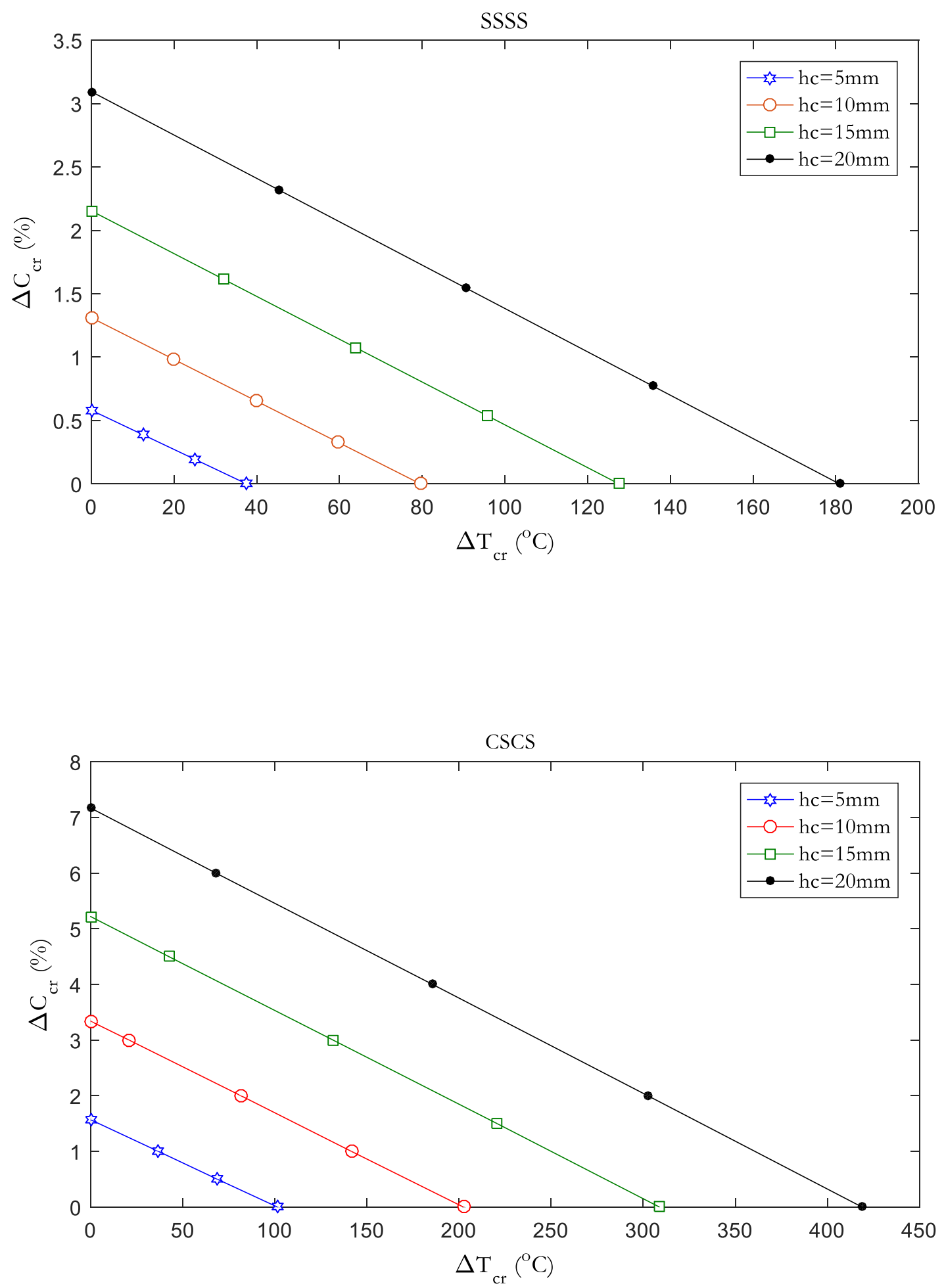


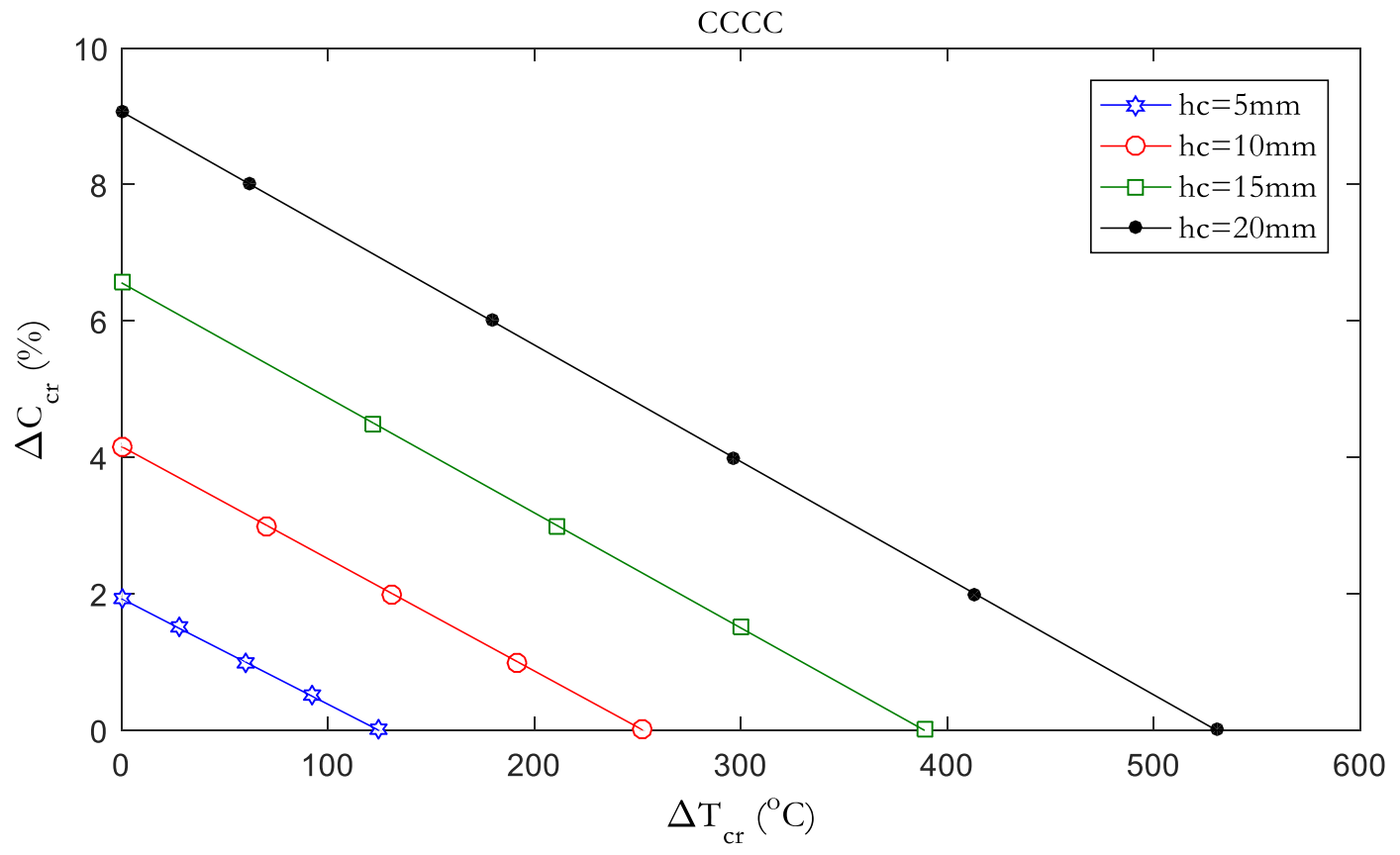

Fig 4 Variations of critical buckling temperature and critical buckling moisture content for different core thickness and various boundary conditions $\left(a=0.4 \mathrm{~m}, b=0.4 \mathrm{~m}, P_{x}=P_{y}=0\right)$ 

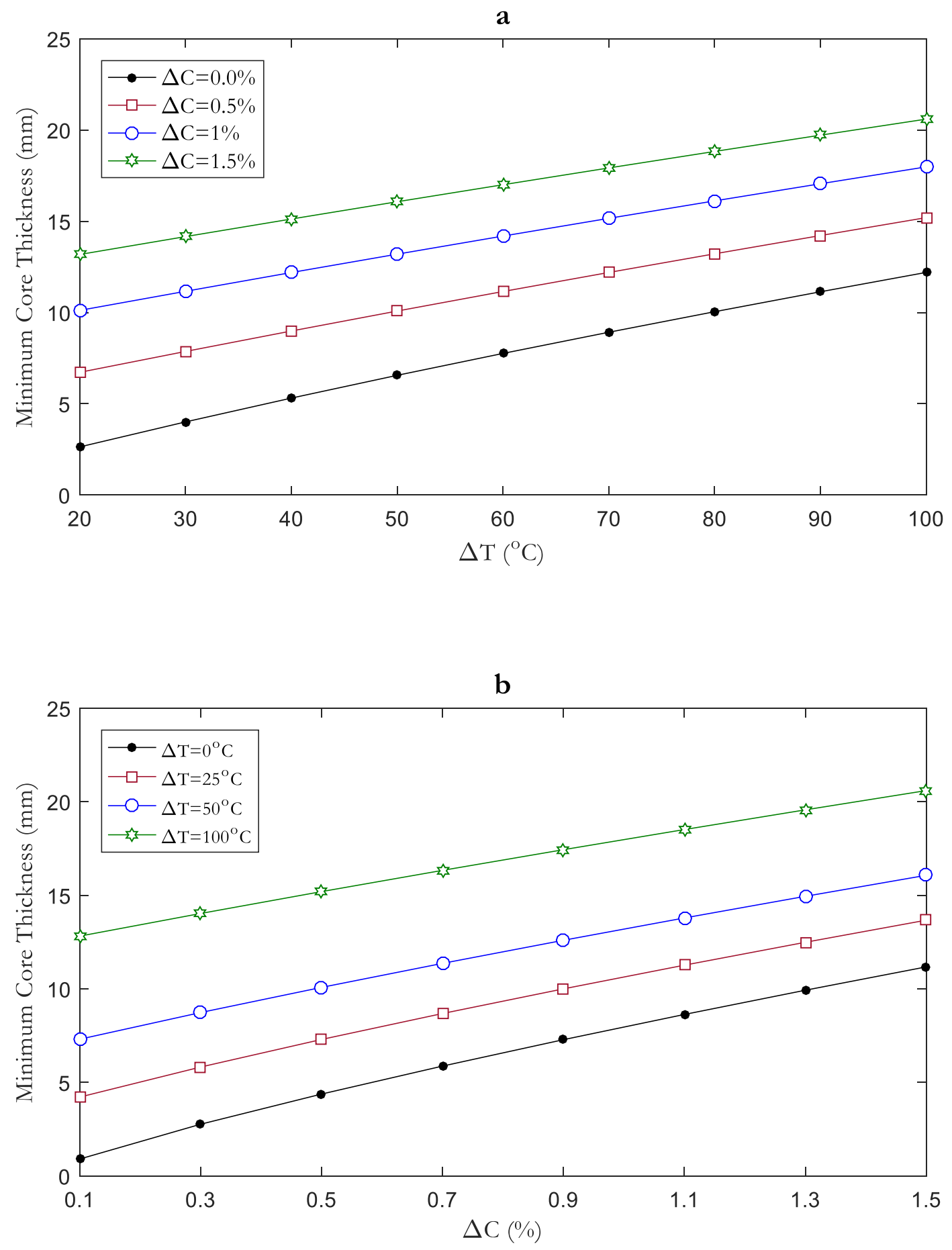

Fig 5 Minimum core thickness required to prevent buckling phenomenon in simply supported sandwich plates due to hygrothermal environment effects $\left(a=0.4 m, b=0.4 m, P_{x}=P_{y}=0\right)$ 


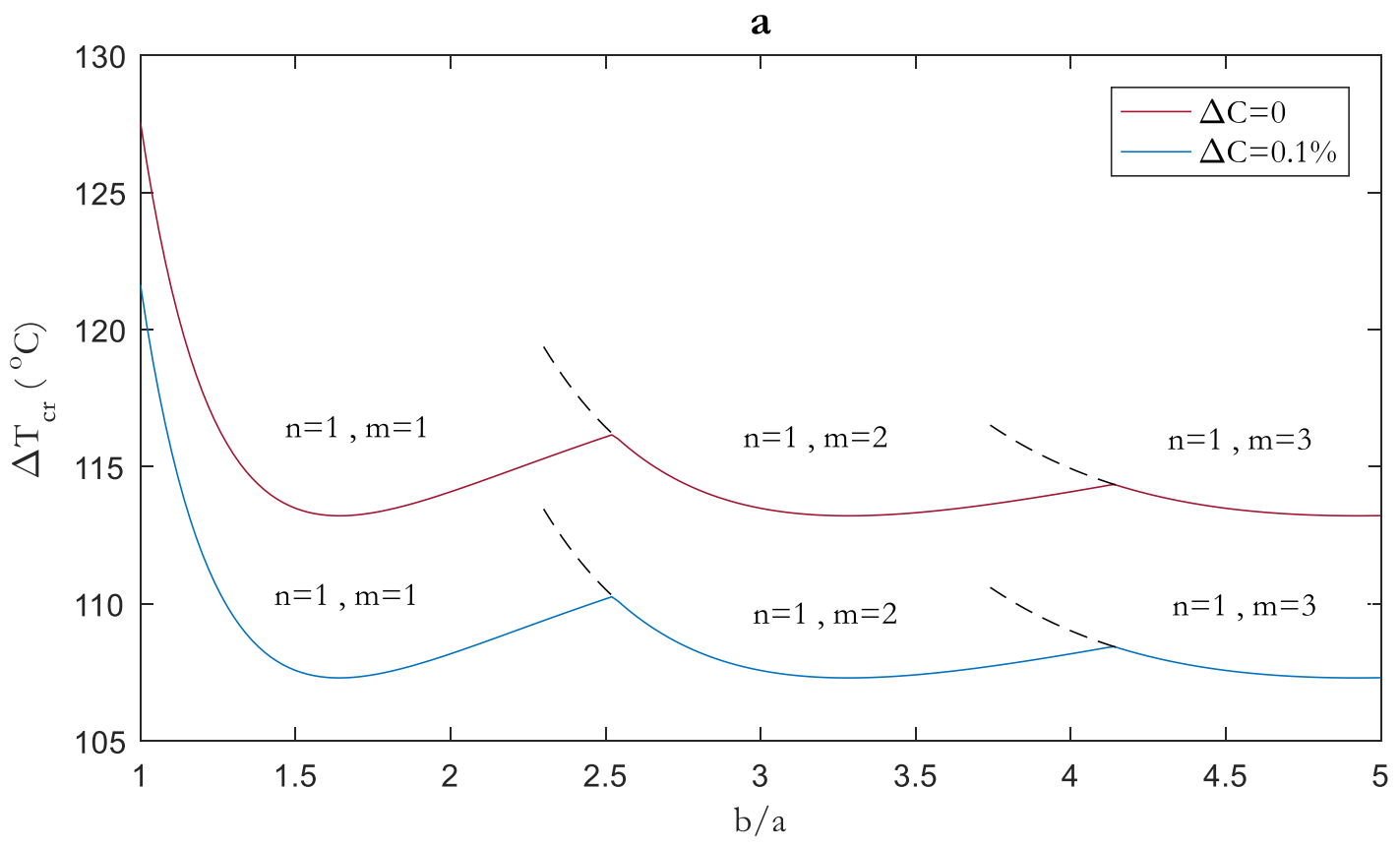

b

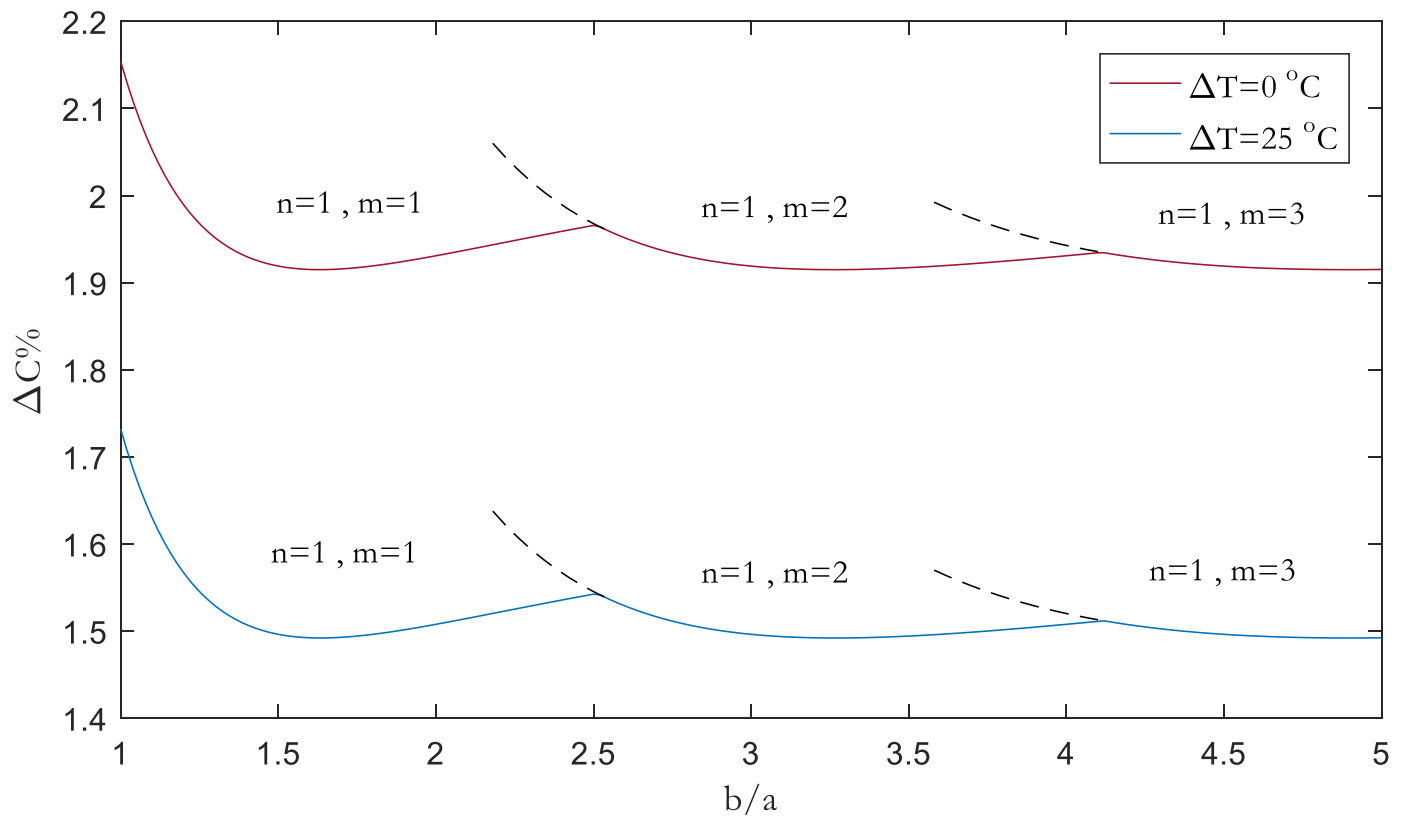

Fig 6 Critical values of temperature and moisture content for hygrothermal buckling of simply supported sandwich plates in the absence of mechanical loads

$$
\left(a=0.4 \mathrm{~m}, h_{c}=15 \mathrm{~mm}, P_{x}=P_{y}=0\right)
$$


$\mathbf{a}$

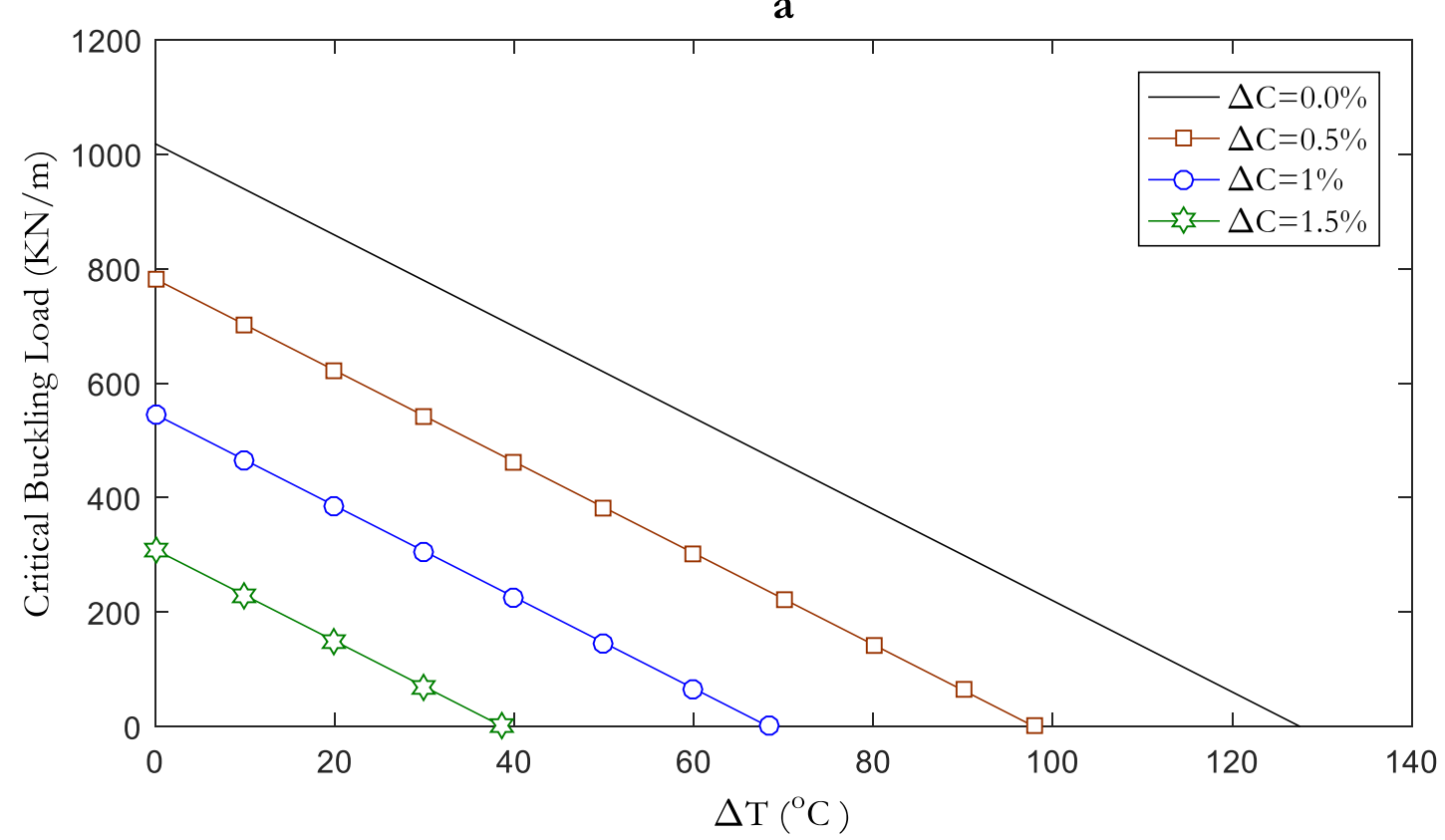

b

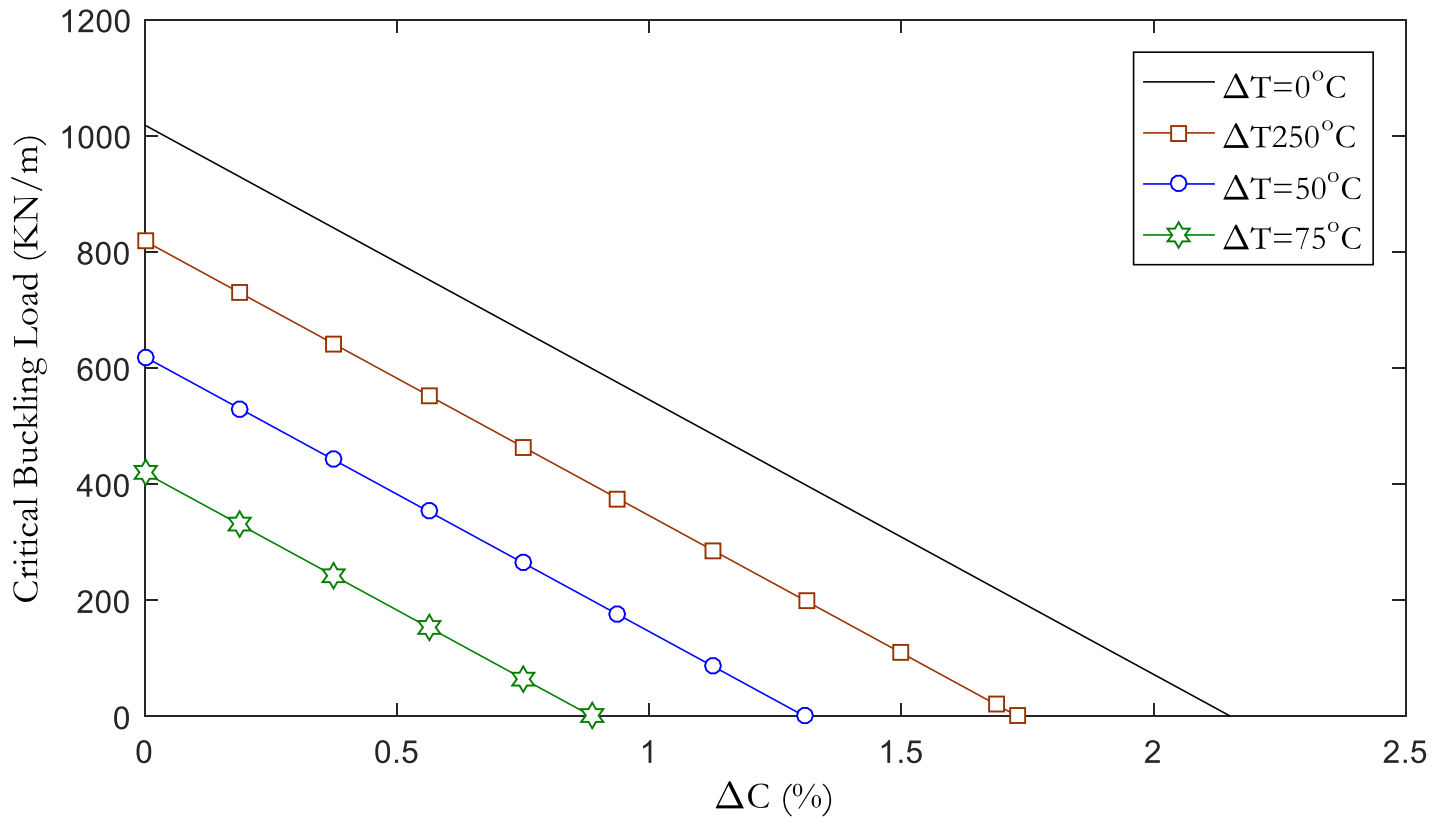

Fig 7 Variations of critical buckling load of sandwich plates with simply supported boundary conditions under uniaxial load and hygrothermal effects $\left(a=b=0.4 \mathrm{~m}, h_{c}=15 \mathrm{~mm}, \lambda=0\right)$ 

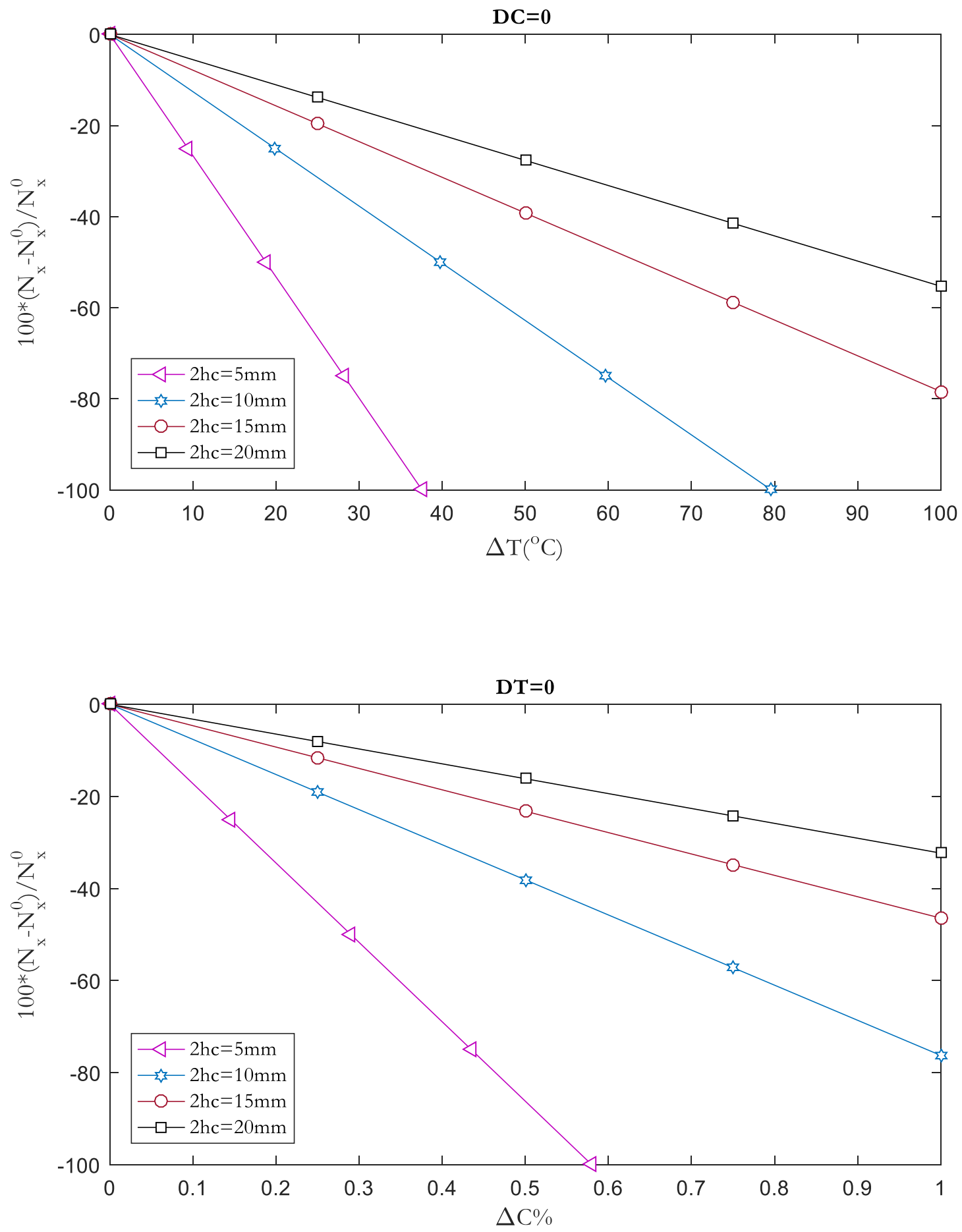

Fig 8 Effects of hygrothermal environment on the critical buckling of sandwich plates with simply supported boundary conditions $(a=b=0.4 m, \lambda=0)$ 

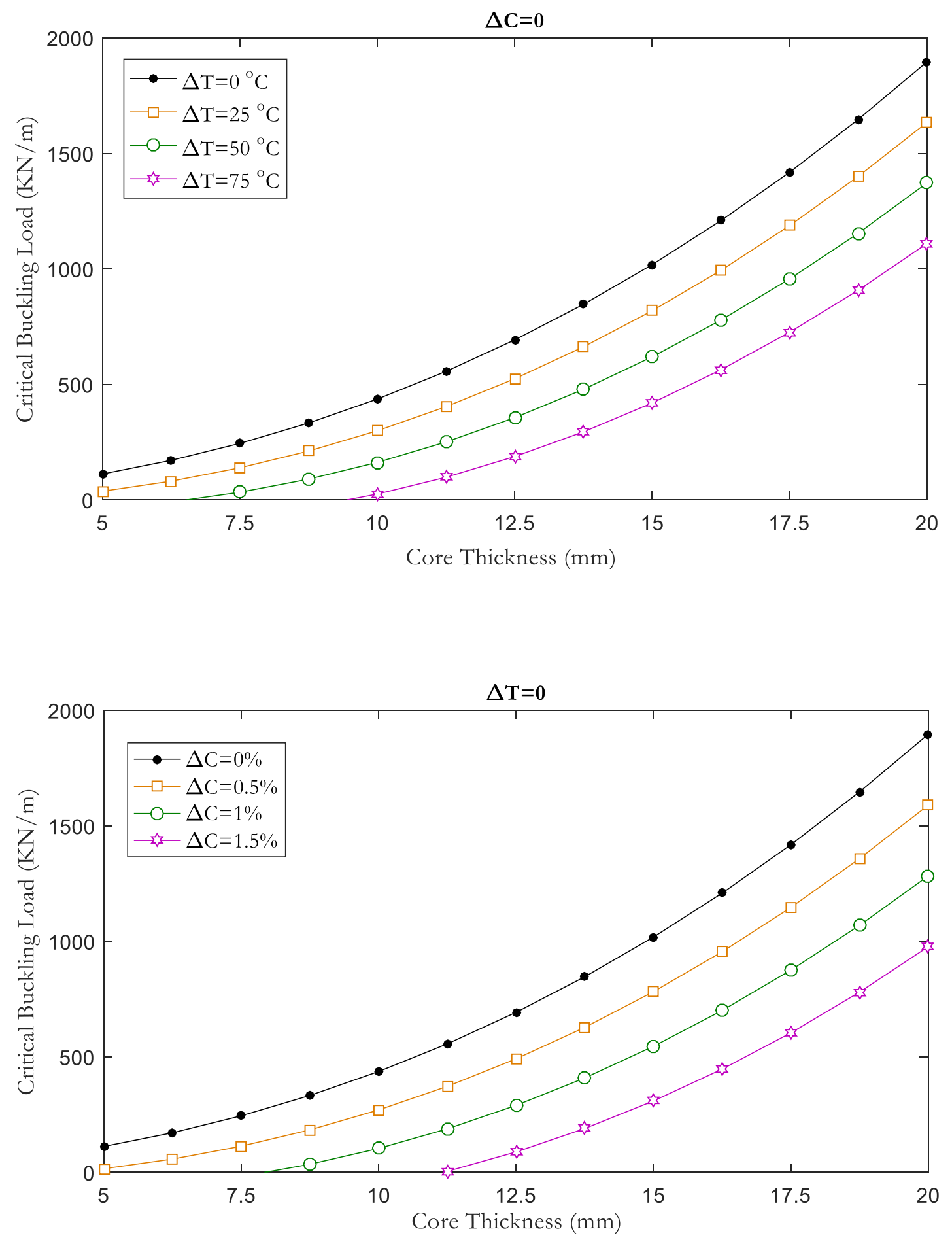

Fig 9 Variations of critical buckling load of simply supported sandwich plates under uniaxial load against core thickness for various hygrothermal conditions $(a=b=0.4 m, \lambda=0)$ 

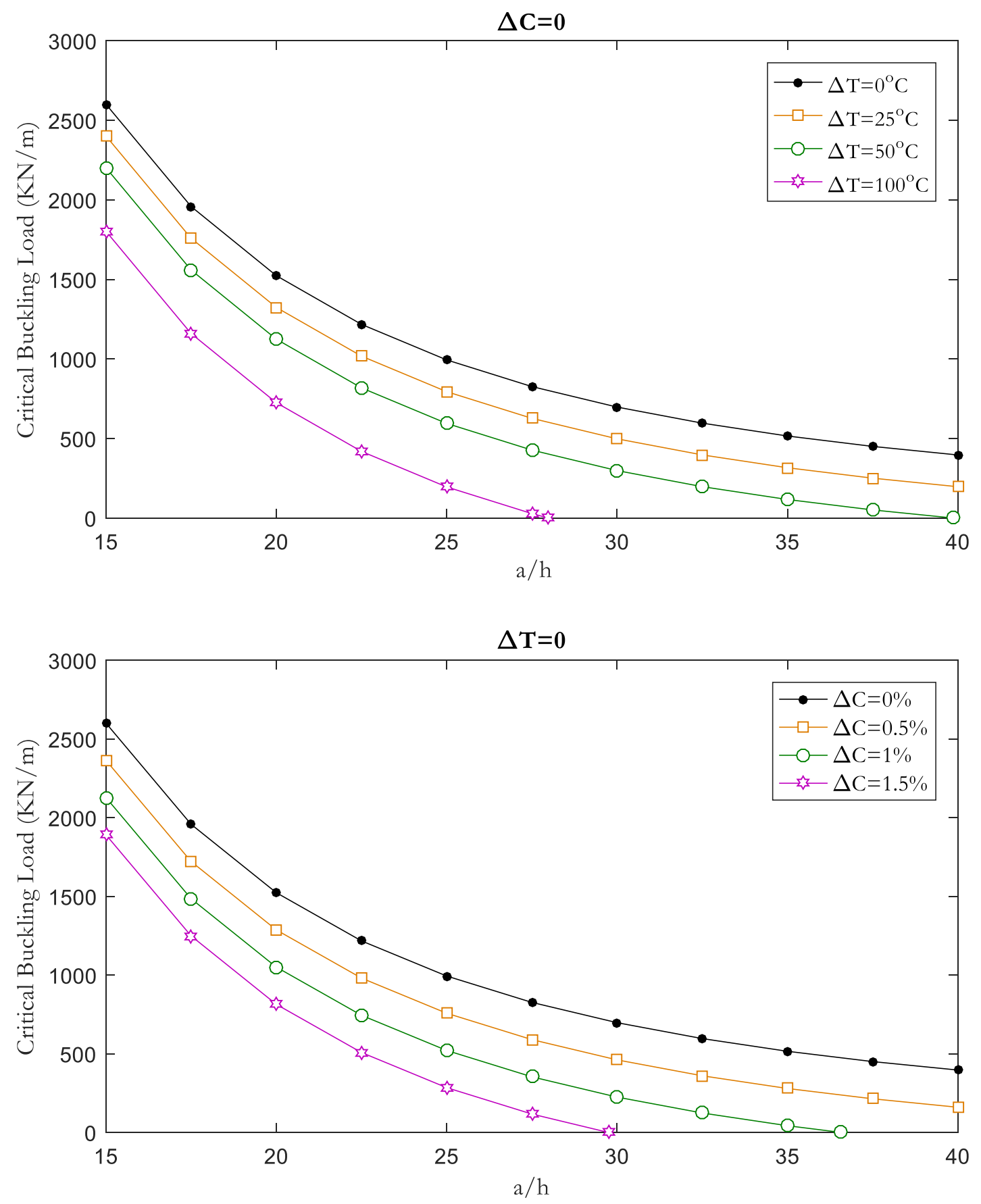

Fig 10 Variations of critical buckling loads of simply supported square sandwich plates versus $a / h$ ratio for a variety hygrothermal conditions $(a=b, h c=15 \mathrm{~mm}, \lambda=0)$ 

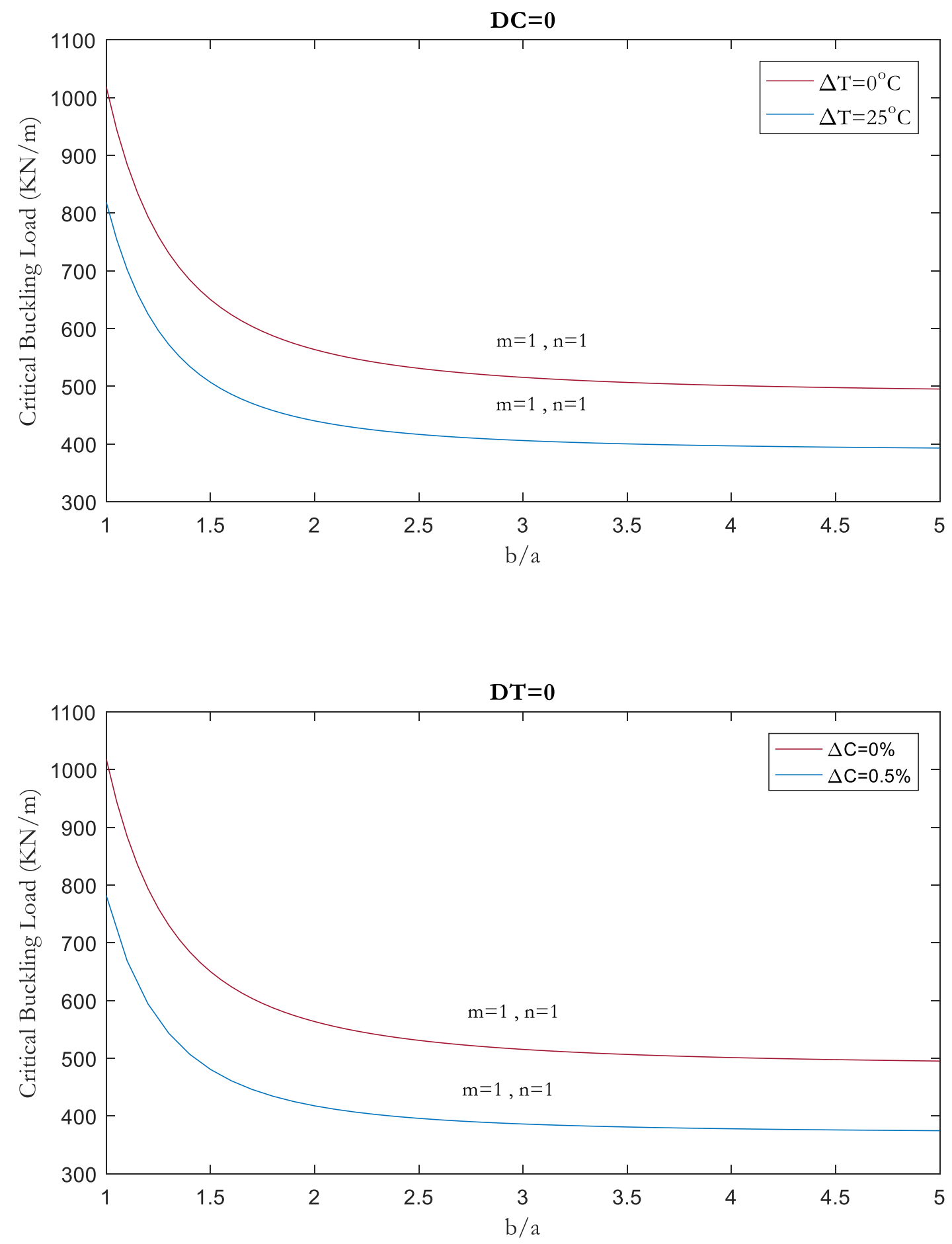

Fig 11 Variations of critical buckling load of simply supported sandwich plates under uniaxial compressive load $(a=0.4 \mathrm{~m}, h c=15 \mathrm{~mm}, \lambda=0)$ 

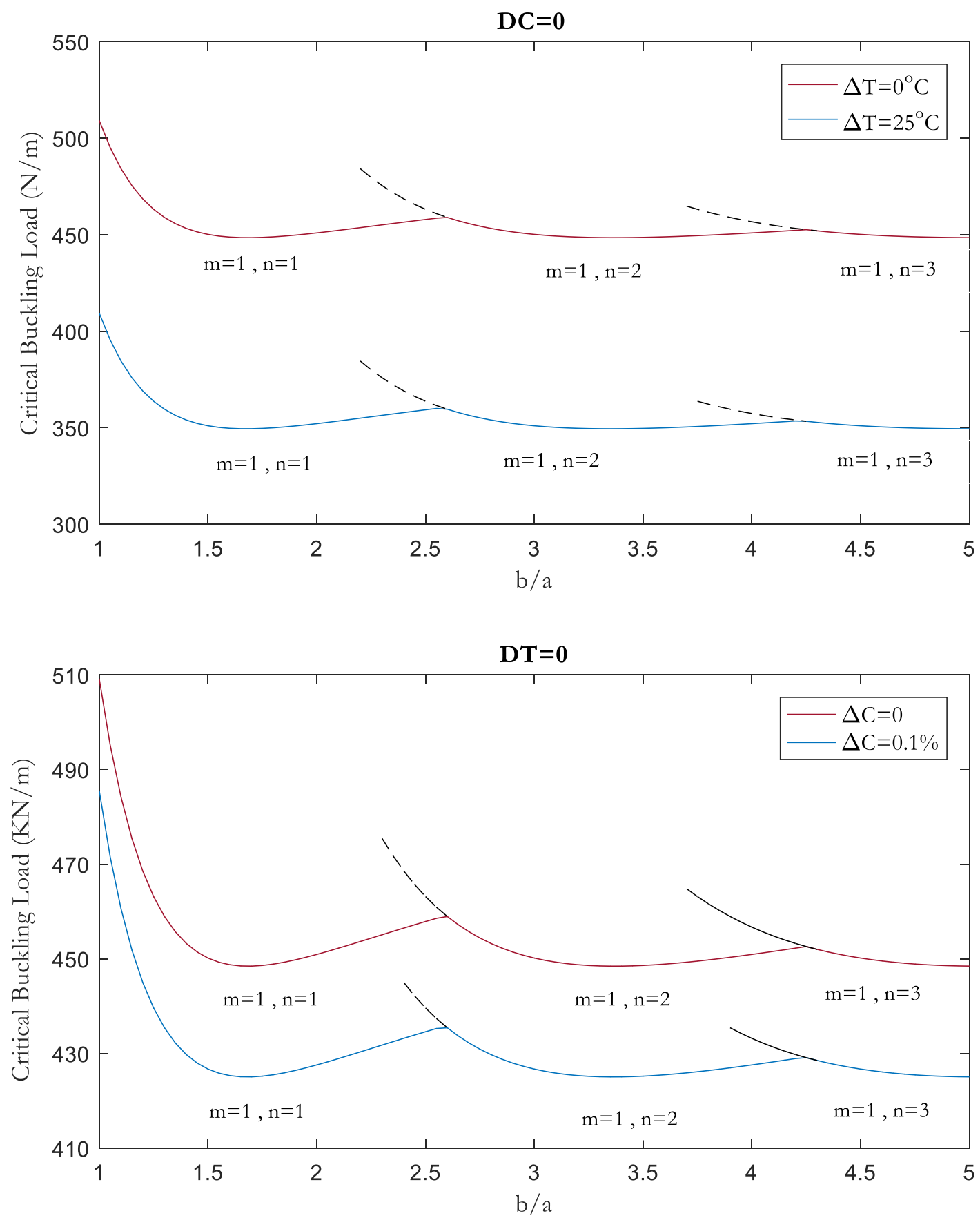

Fig 12 Variations of critical buckling load of simply supported sandwich plates under biaxial compressive load $(a=0.4 \mathrm{~m}, h c=15 \mathrm{~mm}, \lambda=1)$ 
Table 1 Critical buckling stress $\left(\sigma_{c r}=N_{x} / 2 h_{f}\right.$ in $\left.N / \mathrm{mm}^{2}\right)$ of soft-core sandwich plates with isotropic skins under uniaxial $(\lambda=0)$ and biaxial $(\lambda=0.5)$ loads

\begin{tabular}{|c|c|c|c|c|c|c|}
\hline \multirow{2}{*}{ Loading } & \multirow{2}{*}{$\begin{array}{l}\text { Boundary } \\
\text { Conditions }\end{array}$} & \multirow{2}{*}{\multicolumn{2}{|c|}{ References }} & \multicolumn{3}{|c|}{ Aspect ratio $(a / b)$} \\
\hline & & & & 0.5 & 0.7 & 1 \\
\hline \multirow{4}{*}{$\begin{array}{l}\text { Uniaxial compression } \\
(\lambda=0)\end{array}$} & \multirow[t]{4}{*}{ SSSS } & \multirow{2}{*}{\multicolumn{2}{|c|}{$\begin{array}{l}\text { Present (Analytical method) } \\
\text { [46] }\end{array}$}} & 72.080 & 54.332 & 49.003 \\
\hline & & & & 73.305 & 54.924 & 49.373 \\
\hline & & \multicolumn{2}{|l|}{$[47]$} & 73.227 & 54.877 & 49.334 \\
\hline & & \multicolumn{2}{|l|}{ [48] } & 73.1255 & 54.8445 & 49.3192 \\
\hline \multirow{8}{*}{$\begin{array}{l}\text { Biaxial compression } \\
(\lambda=0.5)\end{array}$} & \multirow[t]{8}{*}{$\mathrm{CCCC}$} & \multirow[t]{5}{*}{ Present (GDQ method) } & $(7 \times 7)$ & 170.346 & 111.153 & 80.954 \\
\hline & & & $(9 \times 9)$ & 170.290 & 111.197 & 80.892 \\
\hline & & & $(13 \times 13)$ & 170.286 & 111.153 & 80.890 \\
\hline & & & $(17 \times 17)$ & 170.286 & 111.153 & 80.890 \\
\hline & & & $(21 \times 21)$ & 170.286 & 111.153 & 80.890 \\
\hline & & \multicolumn{2}{|l|}{ [46] } & 170.91 & 112.41 & 81.45 \\
\hline & & \multicolumn{2}{|l|}{ [47] } & 170.11 & 111.15 & 80.95 \\
\hline & & \multicolumn{2}{|l|}{ [48] } & 168.9920 & 110.7877 & 80.7665 \\
\hline
\end{tabular}

Table 2 Critical buckling load ( $N / \mathrm{m}$ ) of simply supported sandwich plates with single-layer face sheets $(\theta /$ Core $/ \theta$ ) under uniaxial compressive loading

\begin{tabular}{lll}
\hline \multirow{2}{*}{ References } & \multicolumn{1}{c}{$\boldsymbol{\theta}$} \\
\cline { 2 - 3 } & $0^{\circ}$ & $90^{\circ}$ \\
\hline Present & 417.03 & 213.67 \\
{$[47]$} & 424.6 & 211.7 \\
{$[49]$} & 423.8 & 213.8 \\
{$[50]$} & 427.6 & 215.6 \\
{$[51]^{*}$} & 424.6 & 214.6 \\
\hline
\end{tabular}

* The data have been obtained through Digitizer software 
Table 3 Verification study for analytical, semi-analytical and numerical methods in critical buckling temperature $\left({ }^{\circ} F\right)$ estimation of sandwich plates with aluminum honeycomb core and titanium face sheets ( $a=24$ in $h=0.81$ in,$h_{f}=0.06$ in $)$

\begin{tabular}{|c|c|c|c|c|c|c|}
\hline \multirow{2}{*}{$\begin{array}{l}\text { Boundary } \\
\text { Conditions }\end{array}$} & \multirow{2}{*}{$b / a$} & \multicolumn{2}{|c|}{ Present } & \multicolumn{2}{|c|}{ [52] } & \multirow{2}{*}{ [28] } \\
\hline & & Solution & & Energy method & Finite element & \\
\hline \multirow[t]{4}{*}{ SSSS } & 1 & Analytical & $627.5(1,1)^{*}$ & 622 & 583 & 631 \\
\hline & 2 & & $409.9(1,1)$ & 409 & 403 & 411 \\
\hline & 3 & & $367.6(1,1)$ & 368 & 366 & 368 \\
\hline & 4 & & $352.6(1,1)$ & 358 & 348 & 353 \\
\hline \multirow[t]{4}{*}{ CSCS } & 1 & Semi-Analytical & $1127.4(1)^{* *}$ & 1128 & 1093 & - \\
\hline & 2 & & $1127.4(2)$ & 1128 & 1096 & - \\
\hline & 3 & & $1127.4(3)$ & 1128 & 1095 & - \\
\hline & 4 & & $1126.8(3)$ & 1126 & 1100 & - \\
\hline \multirow[t]{4}{*}{$\mathrm{CCCC}$} & 1 & Numerical & 1442.0 & 1428 & 1396 & 1456 \\
\hline & 2 & & 1181.2 & 1175 & 1160 & 1173 \\
\hline & 3 & & 1152.0 & 1150 & 1145 & 1148 \\
\hline & 4 & & 1147.2 & 1140 & 1121 & 1136 \\
\hline
\end{tabular}

* The numbers in the parenthesis show half waves numbers $(m, n)$ in Eq. (16) for analytical solution

**The number in the parenthesis denotes half wave number $(n)$ in Eq. (25) for semi-analytical solution

Table 4 Comparative study for critical moisture content (\%) for bucking of a single-layer composite square plate

\begin{tabular}{cccc}
\hline \multirow{2}{*}{ Boundary conditions } & \multirow{2}{*}{ References } & \multicolumn{2}{c}{$\theta$} \\
\cline { 3 - 4 } & Present & 0.434 & $90^{\circ}$ \\
\hline SSSS & {$[22]$} & 0.441 & 0.434 \\
& & & 0.441 \\
SCSC & Present & 0.367 & 0.246 \\
& {$[22]$} & 0.369 & 0.247 \\
CCCC & Present & 0.182 & 0.182 \\
& {$[22]$} & 0.184 & 0184 \\
\hline
\end{tabular}


Table 5 Critical uniaxial buckling load of sandwich plates for various types of boundary conditions

$$
(a=0.4 m, b=0.4 m, \Delta C=0 \%)
$$

\begin{tabular}{|c|c|c|c|c|c|c|}
\hline \multirow{2}{*}{$\begin{array}{c}\text { Boundary } \\
\text { Conditions }\end{array}$} & \multirow[t]{2}{*}{$h_{c}(\mathrm{~mm})$} & \multicolumn{5}{|l|}{$\Delta T\left({ }^{o} C\right)$} \\
\hline & & 0 & 25 & 50 & 75 & 100 \\
\hline \multirow[t]{3}{*}{ SSSS } & 5 & 112.12 & 37.52 & - & - & - \\
\hline & 10 & 436.54 & 299.44 & 162.34 & 25.24 & - \\
\hline & 15 & 1018.4 & 818.81 & 619.20 & 419.60 & 220.00 \\
\hline \multirow[t]{3}{*}{ CSCS } & 5 & 265.22 & 200.50 & 135.35 & 69.789 & 3.856 \\
\hline & 10 & 978.90 & 860.05 & 740.60 & 620.72 & 500.37 \\
\hline & 15 & 2177.8 & 2005.0 & 1831.6 & 1657.6 & 1483.0 \\
\hline \multirow[t]{3}{*}{ CCCC } & 5 & 362.44 & 293.01 & 222.08 & 149.53 & 75.145 \\
\hline & 10 & 1339.6 & 1214.6 & 1088.1 & 960.06 & 830.45 \\
\hline & 15 & 2973.9 & 2795.6 & 2615.7 & 2434.1 & 2250.9 \\
\hline
\end{tabular}

Table 6 Critical uniaxial buckling load of sandwich plates for various types of boundary conditions

$$
\left(a=0.4 m, b=0.4 m, \Delta T=0^{\circ} C\right)
$$

\begin{tabular}{ccccccc}
\hline Boundary & $h_{c}(m m)$ & $\Delta C(\%)$ & & & & \\
\cline { 3 - 6 } Conditions & & 0 & 0.1 & 0.2 & 0.5 & 1 \\
\hline SSSS & 5 & 112.12 & 92.83 & 73.54 & 15.67 & - \\
& 10 & 436.54 & 403.25 & 369.96 & 270.08 & 103.63 \\
& 15 & 1018.4 & 971.11 & 923.82 & 781.95 & 545.50 \\
CSCS & 5 & 265.22 & 248.54 & 231.84 & 181.53 & 97.126 \\
& 10 & 978.90 & 950.10 & 921.27 & 834.59 & 689.54 \\
& 15 & 2177.8 & 2136.9 & 2096.0 & 1973.1 & 1767.6 \\
CCCC & & & & & & \\
& 5 & 362.44 & 344.64 & 326.73 & 272.42 & 179.81 \\
& 10 & 1334.0 & 1309.4 & 1279.1 & 1187.7 & 1033.6 \\
& 15 & 2973.9 & 2931.8 & 2889.6 & 2762.6 & 2549.0 \\
\hline
\end{tabular}

